\title{
SINGULARITIES WITH SYMMETRIES, ORBIFOLD FROBENIUS ALGEBRAS AND MIRROR SYMMETRY
}

\author{
RALPH M. KAUFMANN
}

\begin{abstract}
Previously, we introduced a duality transformation for Euler $G^{-}$ Frobenius algebras. Using this transformation, we prove that the simple $A, D, E$ singularities and Pham singularities of coprime powers are mirror selfdual where the mirror duality is implemented by orbifolding with respect to the symmetry group generated by the grading operator and dualizing. We furthermore calculate orbifolds and duals to other $G$-Frobenius algebras which relate different $G$-Frobenius algebras for singularities. In particular, using orbifolding and the duality transformation we provide a mirror pairs for the simple boundary singularities $B_{n}$ and $F_{4}$. Lastly, we relate our constructions to $r$ spin-curves, classical singularity theory and foldings of Dynkin diagrams.
\end{abstract}

\section{INTRODUCTION}

In [Ka3] we introduced a duality transformation for Euler $G$-Frobenius algebras which are graded Frobenius algebras whose grading operator is realized by the action of a central element. Using this transformation in the setting of isolated singularities with symmetries, we prove that the simple singularities $A, D, E$ and certain Pham singularities are mirror self-dual where the mirror duality is implemented by orbifolding with respect to the symmetry group generated by the grading operator and dualizing. In particular the invariants of the orbifold are $A_{1}$ while the invariants of the dual are the simple singularity of type $A, D, E$ one started out with. Thus orbifolding and dualizing provides a mirror dual pair to the pair $\left(W, A_{1}\right)$ which is naturally associated to $W$, for $W$ one of the simple singularities $A_{n}, D_{n}, E_{6}, E_{7}, E_{8}$. We also show that the same holds true for Pham singularities of co-prime powers.

Furthermore we calculate orbifolds and duals to other $G$-Frobenius algebras which relate different $G$-Frobenius algebras for singularities to each other. We thereby provide more mirror pairs notably mirror pairs for the simple boundary singularities. In particular $\left(\left(B_{n}, I_{2}(4)\right),\left(I_{2}(4), B_{n}\right)\right)$ is obtained by orbifolding and dualizing either $A_{2 n-1}$ or $D_{n+1}$ by $\mathbb{Z} / 2 \mathbb{Z}$ and $\mathbb{Z} / n \mathbb{Z}$. And $\left(\left(F_{4}, I_{2}(4)\right),\left(I_{2}(4), F_{4}\right)\right)$ obtained by orbifolding $E_{6}$ and dualizing with respect to $\mathbb{Z} / 2 \mathbb{Z}$ and $\mathbb{Z} / 3 \mathbb{Z} \times \mathbb{Z} / 2 \mathbb{Z}$.

The invariants of the $G$-Frobenius algebras based on the singularities with symmetries are related to the singularities considered on the orbifold of $\mathbb{C}^{n}$ with respect to the symmetry group while the duals also conjecturally play a role in the analogs of $r$ spin-curves built on quasi-homogenous polynomials of which special types have been studied by [FJR]. For the exact formulation of these conjectures we refer to $\S 5.1$.

The operation of dualizing as defined in [Ka3] was inspired by the representation theory of $N=2$ super-conformal field theory applied to orbifold Landau-Ginzburg models $[\mathrm{IV}]$. Although the background is very elaborate and involves many highly 
complicated concepts, in the special case we are considering all can be stated in terms of $G$-Frobenius algebras or $D(k[G])$ modules and algebras, where $D(k[G])$ stands for the Drinfel'd double of the group ring $k[G] . \quad D(k[G])$ modules are a special type of $G$-graded $G$-modules, namely those, where the $G$-action acts by conjugation on the $G$-grading, cf. [Mo, Ka6, JKK].

We will first review the background for this operation and then comment on its realization on the level of Euler $G$-Frobenius algebras. The reader not inclined to read about physics can thus skip the following two paragraphs and further comments about physics which can be considered as motivation and continue to the purely algebraic part of the paper.

A so-called $(2,2)$ super-conformal field theory has an $N=2$ super-conformal symmetry for both the left and the right movers. This implies that there are four finite rings which are closed under the naive operator product. These rings are called $(c, c),(a, c),(a, a)$ and $(c, a)$ respectively. In terms of the representation theory these rings are given by fields which are annihilated by certain operators or equivalently satisfy certain constraints for their eigenvalues with respect to the operators $J_{0}, \bar{J}_{0}, L_{0}, \bar{L}_{0}$ of the two $N=2$ super-conformal algebras, which are usually called $q, \bar{q}, h$ and $\bar{h}$ respectively. The left $c$ or $a$ stands for left chiral or anti-chiral and the letter $a$ or $c$ on the right for right chiral or right anti-chiral. An element $|\phi\rangle$ is left chiral if $G_{-1 / 2}^{+}|\phi\rangle=0$ or equivalently $h=\frac{q}{2}$. It is called left antichiral if $G_{-1 / 2}^{-}|\phi\rangle=0$ or equivalently $h=-\frac{q}{2}$. Right chiral means that $\bar{G}_{-1 / 2}^{+}|\phi\rangle=$ 0 or equivalently $\bar{h}=\frac{\bar{q}}{2}$ and finally right anti-chiral means that $\bar{G}_{1 / 2}^{-}|\phi\rangle=0$ or equivalently $\bar{h}=-\frac{\bar{q}}{2}$. It turns out the rings $(a, a)$ and $(c, a)$ can be recovered from $(c, c)$ and $(a, c)$ by charge conjugation. Thus one confines oneself to study the latter two rings. Mirror symmetry as it was originally conceived in physics was an operation which takes one conformal field theory $T$ and produces another conformal field theory $\check{T}$ such that the $(c, c)$ ring of $T$ is isomorphic to the $(a, c)$ ring of $\check{T}$ and vice versa.

One special type of $N=2$ theory is given by the so-called Landau-Ginzburg theory which is the conformally invariant fixed point of the Lagrangian

$$
\mathcal{L}=\int K(X, \bar{X}) d^{2} z d^{4} \theta+\int f\left(z_{i}\right)+\text { complex conjugate } d^{2} z d^{2} \theta .
$$

where $f$ is a quasi-homogenous function of fractional degree $q_{i}$ for $z_{i}$. This model leads to a trivial $(a, c)$ ring and a $(c, c)$ ring which is given by $\mathbb{C}[\mathbf{z}] / J_{f}$ where $J_{f}=$ $\left(f_{z_{i}}\right)$ is the Jacobian ideal. Moreover the bi-degree $(q, \bar{q})$ for $z_{i}$ is given by $\left(q_{i}, q_{i}\right)$.

The above considerations are the starting point for a purely algebraic consideration. If the function $f$ above has an isolated singularity at zero, the situation is one that has been studied for a long time by mathematicians. The $(c, c)$ ring is in this case just the local or Milnor ring of the singularity. The only unusual thing is the bi-grading instead of the grading, but in fact the bi-grading is just a diagonal grading obtained from the usual grading in singularity theory and it contains no additional information. It will however play an important role later on.

In the setup above, the quasi-homogeneity of the function $f$ allows one to consider it as a function on a weighted projective space. In the case that the polynomial describes a Calabi-Yau hypersurface the claim these two geometries (singularity/Calabi-Yau) should give the same Frobenius manifolds of field theories is the famous Landau-Ginzburg/Calabi-Yau correspondence. In doing so one 
is naturally considering the quotient of the theory by a finite symmetry group. In general one can consider a group $G \subset G L(\mathbb{C}, n)$ which leaves $f\left(z_{1}, \ldots, z_{n}\right)$ invariant and consider the resulting orbifold. This particular situation and the general setup of global orbifolds was analyzed in [Ka3]. It turns out that the algebraic object one is dealing with is an extension of the Milnor ring, which by itself is a Frobenius algebra, to a $G$-Frobenius algebra in the sense of [Ka3]. A $G$-Frobenius algebra has a $G$-action and the invariants of this $G$-Frobenius are expected to form a Frobenius algebra. These will be bi-graded in a natural way. In physics terms this algebra of invariants is the $(c, c)$ ring of the orbifold model. Now again appealing to physics, the orbifold theory should also have an $(a, c)$ ring. This ring is what is computed by the duality transformation we gave in [Ka3]. To be precise, the ring $(a, c)$ will be equal to the $G$-invariants of the dual $D(k[G])$ model. In order to define the full dual it is necessary for the group of symmetries to contain the symmetry provided by the exponential grading operator $J=\operatorname{diag}\left(\exp \left(2 \pi i q_{1}\right), \ldots \exp \left(2 \pi i q_{n}\right)\right.$.

We called the transformation a mirror transformation, since as we show below, the orbifold of the simple singularities of type $A, D, E$ by the symmetry group generated by $J$ has a trivial $(c, c)$ and an $(a, c)$ ring that is isomorphic to the Milnor ring of the singularity and hence is mirror dual to the original Milnor ring. Thus for these singularities the operation of orbifolding and taking the invariants of the dual (i.e. the $(a, c)$ ring) implements mirror symmetry. If one would like to phrase mirror symmetry in terms of $A$-models and $B$-models, the LandauGinzburg model is a $B$-model. In mathematical terms the $B$-Model is the Milnor ring with the diagonal bi-grading $(q, q)$. The corresponding mirror model is an $A$-model (not to be confused with the $A$-type singularity) which would be given by the Milnor ring but with a grading of $(-q, q)$. This would be a "Landau-Ginzburg A-model".

In [Ka3], we have made the case that for global orbifolds it is not enough to consider just the invariants of the $G$-action of the $G$-Frobenius algebra, but instead one needs to consider the whole $G$ Frobenius. The fruitfulness of this point of view can be seen for instance in its application to symmetric products, [Ka4]. Another instance where the relevance of the $G$-Frobenius algebra is apparent is in the tensor product which exists on the level of $G$-Frobenius algebras and not their invariants. The philosophy extends beyond the level of Frobenius algebras to their deformations, $G$-cohomological field theories as demonstrated in [JKK].

The dualization as we described it in [Ka3] and which we will review below, does not always provide a $G$-Frobenius algebra. In fact generally the data of the $D(k[G])$ model with metric does not afford a $G$-Frobenius algebra structure, although it is expected that there is a Frobenius structure on the invariants. This leads us to define the notion of a degenerate $G$-Frobenius algebra below. Here one adds an additional metric which is equal to the original metric when restricted to the invariants, but is allowed to be degenerate on the non-invariant elements and is invariant w.r.t. a $G$-graded multiplication. This multiplication together with the metric descend to a Frobenius algebra on the invariants.

It is this type of structure that arises in the theory of spin curves [JKV, PV, P] and the construction of cohomological field theories from certain singularities with fixed Abelian groups $H$ containing the grading symmetry $J$, which have recently started to be investigated [FJR]. We conjecture that the resulting theory is the deformation of the dual of the orbifold of the singularity with respect to the group 
$H$. Although the structures coincide on the invariant part, on the degenerate part the matching of non-invariant elements is only almost realized. There are additional elements which can be explained by interpreting the Milnor rings inside degenerate $G$-Frobenius algebras, as we discuss in $\S 5.1$.

In these geometric settings the $g$-twisted sectors - which is another name for the group degree $g$ part of the Frobenius algebra for $g \neq e^{-}$which have a degenerate metric, are related a certain behaviour called of of Ramond type. In the case of the $A_{n}$ singularities there is only one such sector and the entire sector is degenerate. In the cases of $D$ and $E$, the structure is more complicated and there are invariant elements in $g$-twisted sectors which have a degenerate metric. The appearance of these degenerate elements is stunning and maybe a nuisance from the point of view of spin-curves, but is natural from the $G$-Frobenius point of view. Moreover regarding our dualization on the level of $G$-Frobenius algebras as mirror symmetry, we expect this kind of behavior for the mirror dual " $A$-model" of a singularity, the construction of which was Witten's original motivation for considering the spincurve picture $[\mathrm{W}]$.

A note of caution about nomenclature. One would be inclined to call the sectors having degenerate pairings in the new metric Ramond sectors. This might however lead to confusion, since the term Ramond already has a meaning in the theory of $G$-Frobenius algebras [Ka3] and orbifold Landau-Ginzburg theory. Therefore we will call them sectors of Ramond type and hope to avoid the confusion.

We recall that the Ramond $G$ algebra or state-space for a $G$-Frobenius algebra is a cyclic module for the $G$-Frobenius algebra whose $G$-action is determined by compatibility and the fact that the generator of the cyclic algebra is the one dimensional representation of $G$ which is given by the character $\chi$ which is part of the data of a $G$-Frobenius algebra. The component of this space of group degree $g$ would be naturally called the $g$-twisted Ramond sector. The Ramond in this name stand for the Ramond ground states. This Ramond space plays a fundamental role in the theory of singularities as it corresponds as a $D(k[G])$ module to the middle dimensional cohomology of the Milnor fibers in an orbifold model, while the $G^{-}$ Frobenius algebra corresponds as a $D(k[G])$ module to the orbifold Milnor ring or universal deformation space. (See the remarks in $\S 5.2$ below). For the untwisted sectors, i.e. the subalgebras of group degree $e$, this statement was first proved in [Wa].

In the sprit of the mirror construction for simple singularities one expects that for a given theory $T$ with a symmetry group $G$ and a subgroup $H \subset G$ of symmetries $(T / H)^{H} \simeq\left(((T / H) /(G / H))^{\vee}\right)^{(G / H)}$ where the subscript stands for taking the invariants and $\vee$ stands for dualizing. This type of transformation was used by [GP] to produce the first mirror pairs. The general statement has to be taken as always cum grano salis, but as we show below it is true in many instances.

Lastly the untwisted sector of an orbifold associated to a singularity can under certain conditions be related to the folding of an associated Dynkin diagram. We emphasize that there are foldings and orbifoldings of diagrams. The $\mathbb{Z} / 2 \mathbb{Z}$ folding of $A_{2 n-1}$ yields $B_{n}$ while the $\mathbb{Z} / 2 \mathbb{Z}$ orbifolding yields $D_{n+1}$.

In order to understand the operation of folding, we also include section 5.3 in this paper on groups of projective symmetries. This is a new construction for Frobenius algebras which we expect to be able to extend to the respective Frobenius manifolds and to a full theory of $G$-Frobenius algebras. On the algebra level, we obtain the 
classical folding results for Coxeter groups identifying the sub-Frobenius algebra with the Coxeter group of the folded diagram [St]. The relation to singularity theory and the Milnor fibration is also briefly discussed. One could hope to extend the folding to all the diagrams of $[\mathrm{Z}]$ and find the corresponding orbifold theory.

We will work in the setting of $G$-Frobenius algebras over a field $k$ of characteristic zero (or prime to $|G|$ ) as it was established in [Ka3]. To understand the constructions of $G$-Frobenius algebra it is important to see that they are usually performed in four steps. 1. One constructs a $G$-graded $k$-module $A=\bigoplus A_{g}$ with a non-degenerate paring between $A_{g}$ and $A_{g^{-1}}$ and together with an $A_{e}$ module structure on $A$. $A_{e}$ is usually called the untwisted sector and $A_{g}$ is called the $g-$ twisted sector. 2. One constructs a $D(k[G])$ module structure on $A$ compatible with the $A_{e}$ module structure. I.e. one gives an action of $G$ together with a character $\chi \in \operatorname{Hom}\left(G, k^{*}\right) \varphi$ s.t. $\varphi(g)\left(A_{h}\right) \subset A_{g h g^{-1}}$ which satisfies the so-called restricted trace condition and the self-invariance for the twisted sectors. 3. Lastly one adds a $G$ multiplication to make the $D(k[G])$ module into a $G$-Frobenius algebra. Comparing $G$-Frobenius algebras on different levels of this construction compares to the topological mirror symmetry of dimensions and vector spaces vs. that of full Frobenius manifolds.

These are also the steps of the (re)construction program as explained in [Ka3, Ka4]. Here the data for the first step is usually provided by the geometric setup. For the second step there are usually several different choices. This is, however, expected, since there is the phenomenon of discrete torsion for orbifolds. As we demonstrated in [Ka6] for every $G$-Frobenius algebra there exists a family of $G^{-}$ Frobenius algebras indexed by elements of $\alpha \in Z^{2}\left(G, k^{*}\right)$ with the same underlying data as mentioned in step 1 (up to a re-scaling of the metrics pairing the twisted sectors). In the last step there is an additional compatibility condition of the pairing, which might force one to again re-scale the pairings between the twisted sectors. For all the conditions, we refer to [Ka3]. We will however review the construction for singularities with symmetries below.

The dualization is an involution on triples $(A, j, \chi)$ of a $D(k[G])$ module $A$, an element $j \in Z(G)$ the center of $G$ and a one-dimensional representation of $G$, also known as a character. For a special type of graded $G$-Frobenius algebras $\chi$ is part of the data while $j$ corresponds to the grading operator. If one includes the other structures of a $G$-Frobenius algebra, then the operation ceases to be an involution as for instance the metric will be compatible with the group grading only up to a shift. To compensate the different behavior of the duals, we introduce the notion of a degenerate $G$-Frobenius algebra of a group degree $j$ for an element $j \in Z(G)$.

The paper is organized as follows: In the first section, we review the construction and basic properties of $G$-Frobenius algebras and consider special types of graded $G$-Frobenius algebras called Euler and $G$-Euler. The second section contains the definition for the dualization for Euler $D(k[G])$ modules. The third section applies the first two sections to the $G$-Frobenius algebras resulting from quasi-homogenous polynomials in general. The fourth section contains explicit calculations for a large list of examples. From these examples we obtain the theorem about the mirror-self duality of the simple singularities, i.e. those of ADE type and the Pham singularities for coprime powers. The examples also provide mirror pairs for the simple boundary singularities $B_{n}$ and $F_{4}$ and produces $G_{2}$ as the untwisted sector of a $D_{4}$ orbifold. 
In the last section, we connect our calculations to spin-curves, classical results in the theory of singularities and foldings of Dynkin diagrams.

\section{ACKNOWLEDGEMENTS}

We would like to thank the organizers of the AMS special session "GromovWitten Theory of Spin Curves and Orbifolds" Tyler Jarvis, Takashi Kimura and Arkady Vaintrob for doing such an excellent job in providing a common platform for all the exciting research revolving around spin curves and orbifolds.

It is also a pleasure to thank Boris Dubrovin, Claus Hertling, Tyler Jarvis, Takashi Kimura, Yongbin Ruan, Ian Strachan, Kyoji Saito, and Jean-Bernard Zuber for discussions which were relevant for various stages of the presented research.

\section{Graded $G$-Frobenius algebras}

1.1. $G$-Frobenius algebras. We would like to recall the definition of a $G$-Frobenius algebra of [Ka3]. Although it has now appeared in many places we think it convenient for the reader to display it here once more.

Definition 1.1. A $G$-Frobenius algebra (FA) over a field $K$ of characteristic 0 is given by the data $\langle G, A, \circ, 1, \eta, \varphi, \chi>$, where

$G$ finite group

$A$ finite dim $G$-graded $K$-vector space

$A=\oplus_{g \in G} A_{g}$

$A_{e}$ is called the untwisted sector and

the $A_{g}$ for $g \neq e$ are called the twisted sectors.

- a multiplication on $A$ which respects the grading:

$\circ: A_{g} \otimes A_{h} \rightarrow A_{g h}$

1 a fixed element in $A_{e}$-the unit

$\eta \quad$ non-degenerate bilinear form

which respects grading i.e. $\left.g\right|_{A_{g} \otimes A_{h}}=0$ unless $g h=e$.

$\varphi$ an action of $G$ on $A$ (which will be by algebra automorphisms),

$\varphi \in \operatorname{Hom}(G, \operatorname{Aut}(A))$, s.t. $\varphi_{g}\left(A_{h}\right) \subset A_{g h g^{-1}}$

$\chi \quad$ a character $\chi \in \operatorname{Hom}\left(G, K^{*}\right)$

Satisfying the following axioms:

Notation: We use a subscript on an element of $A$ to signify that it has homogeneous group degree -e.g. $a_{g}$ means $a_{g} \in A_{g}$ and we write $\varphi_{g}:=\varphi(g)$ and $\chi_{g}:=\chi(g)$.

a) Associativity

$$
\left(a_{g} \circ a_{h}\right) \circ a_{k}=a_{g} \circ\left(a_{h} \circ a_{k}\right)
$$

b) Twisted commutativity

$$
a_{g} \circ a_{h}=\varphi_{g}\left(a_{h}\right) \circ a_{g}
$$

c) $G$ Invariant Unit:

$1 \circ a_{g}=a_{g} \circ 1=a_{g}$

and

$\varphi_{g}(1)=1$ 
d) Invariance of the metric:

$$
\eta\left(a_{g}, a_{h} \circ a_{k}\right)=\eta\left(a_{g} \circ a_{h}, a_{k}\right)
$$

i) Projective self-invariance of the twisted sectors

$$
\varphi_{g} \mid A_{g}=\chi_{g}^{-1} i d
$$

ii) $G$-Invariance of the multiplication

$$
\varphi_{k}\left(a_{g} \circ a_{h}\right)=\varphi_{k}\left(a_{g}\right) \circ \varphi_{k}\left(a_{h}\right)
$$

iii) Projective $G$-invariance of the metric

$$
\varphi_{g}^{*}(\eta)=\chi_{g}^{-2} \eta
$$

iv) Projective trace axiom

$\forall c \in A_{[g, h]}$ and $l_{c}$ left multiplication by $c:$

$$
\chi_{h} \operatorname{Tr}\left(\left.l_{c} \varphi_{h}\right|_{A_{g}}\right)=\chi_{g^{-1}} \operatorname{Tr}\left(\left.\varphi_{g^{-1}} l_{c}\right|_{A_{h}}\right)
$$

We sometimes denote by $\rho \in A_{e}$ the element dual to $\epsilon \in A_{e}^{*}$ and Poincaré dual to $1 \in A_{e}$.

For the examples in $\S 4$ it is essential that we consider $G$-Frobenius algebras with non-trivial characters.

Remark 1.1. Instead of using a left action of $G$ on $A$ one can also use a right action as for instance is done in e.g. [JKK]). Since if $\varphi$ is a left action $\rho(g):=$ $\varphi\left(g^{-1}\right)$ is a right action, it does not matter which choice is made.

Remark 1.2. Another way to characterize a the $G$-grading and $G$-action it to say that it is a $D(k[G])$ module. This statement is equivalent to saying that $A$ is $G$-graded and the $G$-action is such that $(*) \varphi(g) A_{h} \subset A_{g h g^{-1}}$ or $\rho(g) A_{h} \subset A_{g^{-1} h g}$, cf. e.g. [Ka6]. We use the nomenclature of $D(k[G])$ module, rather than $G-$ graded $G$-module since it includes the condition (*).

The compatibilities of the multiplication with the grading and the $G$-action can also be rephrased as $A$ is a $D(k[G])$ module algebra.

1.2. Restriction. The operation of restriction a $G$-Frobenius algebra to a $H$ Frobenius algebra for a subgroup $H \subset G$ is discussed in [Ka3] and is given by $\operatorname{res}(A)_{H}^{G}:=$ $\bigoplus_{h \in H} A_{h}$ and restricting all structures.

By forgetting or omitting the multiplicative structure and considering just the action of the subgroup $H$ we obtain the restriction from a $D(k[G])$ to an $D(k[H])$ module.

1.3. Super-grading. We also need to enlarge the framework by considering superalgebras rather than algebras. This will introduce the standard signs.

Definition 1.2. A G-twisted Frobenius super-algebra over a field $K$ of characteristic 0 is $<G, A, \circ, 1, \eta, \varphi, \chi>$, where 
$G$ finite group

$A$ finite dimensional $\mathbb{Z} / 2 \mathbb{Z} \times G$-graded $K$-vector space

$A=A_{0} \oplus A_{1}=\oplus_{g \in G}\left(A_{g, 0} \oplus A_{g, 1}\right)=\oplus_{g \in G} A_{g}$

$A_{e}$ is called the untwisted sector and is even.

The $A_{g}$ for $g \neq e$ are called the twisted sectors.

- a multiplication on $A$ which respects both gradings:

$\circ: A_{g, i} \otimes A_{h, j} \rightarrow A_{g h, i+j}$

1 a fixed element in $A_{e}$-the unit

$\eta \quad$ non-degenerate even bilinear form

which respects grading i.e. $\left.g\right|_{A_{g} \otimes A_{h}}=0$ unless $g h=e$.

$\varphi$ an action by even algebra automorphisms of $G$ on $A$,

$\varphi \in \operatorname{Hom}_{K-a l g}(G, A)$, s.t. $\varphi_{g}\left(A_{h}\right) \subset A_{g h g^{-1}}$

$\chi \quad$ a character $\chi \in \operatorname{Hom}\left(G, k^{*}\right)$

satisfy the axioms $a)-d$ ) and $i$ )-iii) of a G-Frobenius algebra with the following alteration:

$\mathrm{b}^{\sigma}$ ) Twisted super-commutativity

$$
a_{g} \circ a_{h}=(-1)^{\tilde{a}_{g} \tilde{a}_{h}} \varphi_{g}\left(a_{h}\right) \circ a_{g}
$$

$\left.\mathrm{iv}^{\sigma}\right)$ Projective super-trace axiom

$\forall c \in A_{[g, h]}$ and $l_{c}$ left multiplication by $c$ :

$\chi_{h} \mathrm{~S} \operatorname{Tr}\left(\left.l_{c} \varphi_{h}\right|_{A_{g}}\right)=\chi_{g^{-1}} \mathrm{~S} \operatorname{Tr}\left(\left.\varphi_{g^{-1}} l_{c}\right|_{A_{h}}\right)$

where $\mathrm{STr}$ is the super-trace.

\subsection{Graded $G$-Frobenius algebras.}

Definition 1.3. We call a (super) $G$ Frobenius algebra $A$ graded by an additive group $I$ if it is graded as a (super) algebra by $I$ and the metric is homogenous of a fixed degree $d$, i.e. for homogenous $a, b, \eta(a, b)=0$ unless $\operatorname{deg}(a)+\operatorname{deg}(b)=d$, where we denote the $I$ degree of a homogenous element $a \in A$ by $\operatorname{deg}(a)$. If $I=\mathbb{Q}$, we simply call $A$ graded. We also call $d$ the degree of the Frobenius algebra.

The degree of the Frobenius algebra is the degree of the element $\rho$.

1.5. The grading operator. Given a graded $G$-Frobenius algebra $A$, we define the grading operator $Q$ to be given by

$$
Q(a):=\operatorname{deg}(a) a \text { if } a \text { is homogeneous }
$$

Sometimes this type of operator is also called $E$.

In the case that $A$ is graded and $k=\mathbb{C}$ or $k$ is of characteristic 0 and an embedding of $\bar{k} \subset \mathbb{C}$ has been fixed we furthermore define the operator

$$
J:=\exp (2 \pi i Q)
$$

Definition 1.4. We call a graded $D(k[G])$-module $A=\bigoplus_{g \in G} A_{g}$ Euler if the operator $\left.J\right|_{A_{e}}$ is described by the action of a central element $j$ of the group $G$ on $A_{e}$. I.e. there exists a $j \in Z(G)$, the center of $G,\left.\varphi\left(h^{-1} j\right)\right|_{A_{h}}=\left.J\right|_{A_{h}}$.

We call a graded $D(k[G])$-module $G$-Euler if there exists a $j \in Z(G)$, s.t. $\left.\varphi\left(h^{-1} j\right)\right|_{A_{h}}=\left.J\right|_{A_{h}}$. 
We call a graded $k[D(H)]$ A quasi-Euler (or quasi-G-Euler) if there is a group $G$, s.t. $H$ is a subgroups of $G(G \supset H)$ and there exists an Euler (or $G$-Euler) $D(k[G])$ module $B$ s.t. the restriction of the $D(k[G])$ module $B$ to its $k[D(H)]-$ sub

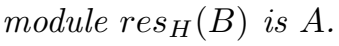

An Eulerization of a quasi-Euler $D[k(H)]$ module is a fixed choice of $D(k[G])$ module $B$ as above.

A $G$-Frobenius algebra is called Euler, $G$-Euler, quasi-Euler or quasi-G-Euler if its underlying $D(k[G])$ module is Euler, $G$-Euler, quasi-Euler or quasi-G-Euler, respectively.

\subsection{Bi-Graded $G$-Frobenius algebras.}

Definition 1.5. We call a (super) $G$ Frobenius algebra $A$ bi-graded by an additive group $I$ if it is bi-graded as a (super) algebra by $I$. If $I=\mathbb{Q}$, we simply call $A$ bi-graded.

1.6.1. Notation. We will usually denote the two grading operators by $Q$ and $\bar{Q}$. Given a bi-homogenous element $a$ we will denote its degree w.r.t. $Q$ by $q(a)=Q(a)$ and its degree w.r.t. $\bar{Q}$ by $\bar{q}(a)=\bar{Q}(a)$. We will also use the notation $(q(a), \bar{q}(a))$ to denote the bi-degree.

Definition 1.6. Fix a graded $G$-Frobenius algebra $A$ with grading operator $\mathcal{Q}$.

We define its $(c, c)$ realization $A^{(c, c)}$ to be given by the $G$-Frobenius algebra $A$ together with the bi-grading $(\mathcal{Q}, \mathcal{Q})$, i.e. $\overline{\mathcal{Q}}=\mathcal{Q}$.

We define the $(a, c)$ realization of $A$ denoted by $A^{(a, c)}$ to be given by the $G-$ Frobenius algebra $A$ together with the bi-grading $(\mathcal{Q},-\mathcal{Q})$, i.e. $\overline{\mathcal{Q}}=-\mathcal{Q}$.

Remark 1.3. The terminology stems from the representation theory of the $N=2$ super-conformal algebra, as explained in the introduction.

1.7. Constructing $G-$ Frobenius algebras. When constructing $G$-Frobenius algebras form geometric or algebraic data usually the different structures are introduced one after the other. A good example of this procedure is given by the construction of $G$-Frobenius algebras from isolated singularities with symmetries reviewed below 3 . Also some operations like the duality discussed below are given on a certain level of structure. The usual order in which the structures are introduced is as follows.

(1) The $G$-graded $k$-module. Usually the first structure to be given for any $G$-Frobenius algebra is its additive structure $A:=\bigoplus_{g \in G} A_{g}$.

On this level it is also usual to introduce the non-degenerate pairing $\eta$ which pairs $A_{g}$ with $A_{g^{-1}}$.

(2) The $G$-graded $G$-module or $D(k[G])$ module structure. The next property which is usually introduced is a $G$-action on $A$, usually denoted by $\varphi$ for a left action (cf. e.g. [Ka3]) which makes $A$ into a $D(k[G])-$ module cf. 1.2 .

Further data and conditions:

(a) Along with the $G$-action the function $\chi: G \rightarrow k^{*}$ is fixed since $\chi$ can be derived from the $G$-action via the condition of projective selfinvariance (axiom ii ${ }^{\sigma}$ ).

(b) From the projective $G$-invariance of the metric (axiom iii), it follows that the function $\chi^{2}$ has to be a character, i.e. a one-dimensional representation. 
(3) The $D(k[G]), A_{e}-\mathbf{b i}-$ module Usually the untwisted sector $A_{e}$ is naturally a Frobenius algebra. The next step in constructing a $G$-Frobenius algebra is then the $A_{e}$-module structure for each $A_{g}: A_{e} \otimes A_{g} \rightarrow A_{g}$, which will be a part of the algebra multiplication. These operations turn $A$ into an $A_{e}$ module. They are usually already present in the geometry by functoriality [Ka4]. The $A_{e}$ module structure has be compatible with the $G$-action so: $\varphi(g)\left(a_{e} b_{h}\right)=\varphi(g)\left(a_{g}\right) \varphi_{g}\left(b_{h}\right)$.

The $A_{e}$ module structure leads to a second compatibility condition of the $G$-action with the pairing which is given by restriction of the trace axiom to the case $g=e, c=1 \in A_{e}$. This condition effectively relates the dimension of the various twisted sectors $A_{g}$ to the character $\chi$ and $G$-action on the identity sector.

$$
\left.\chi_{h} \operatorname{STr} \varphi(h)\right|_{A_{e}}=\left.\operatorname{STr} i d\right|_{A_{h}}=\operatorname{sdim}\left(A_{h}\right)
$$

Also the trace axiom put constraints on the possible $G$-actions. The constrains can be quite effective, but they define the action at most up to discrete torsion [Ka6].

(4) The $G$-Frobenius algebra. The last step is to introduce the stringy multiplications: $A_{g} \otimes A_{h} \rightarrow A_{g h}$, ie. the algebra structure. This structure has to be compatible with the $G$-action and the metric.

1.8. The metric and the grading. When constructing a $G$-Frobenius algebra in the above fashion, the metric and the grading can either be introduced at the end, but usually, there is a natural choice in each step, which may be modified in the next step.

1.8.1. The metric. The metric, i.e. non-degenerate even symmetric pairing, is usually introduced in step (1) and may be re-scaled in step (4) by a factor to ensure the compatibility of the metric with the multiplication (invariance of the metric axiom d)).

1.8.2. The grading. In step (1) first there is usually a grading $Q^{(1)}$ inherent in the definition of each $A_{g}$ when introducing the metric which is usually also inherent in the construction. Each of the pairings $A_{g} \otimes A_{g^{-1}} \rightarrow k$ is usually homogenous of some fixed degree $d_{g}$ with $d_{g}=d_{g^{-1}}$.

The first alteration of the grading is a shift of the grading for each $A_{g}$ by $\frac{1}{2} s^{+}(g):=\frac{1}{2}\left(d_{e}-d_{g}\right)$, i.e. the new grading for an element $a_{g} \in A_{g}$ is $Q^{(2)}\left(a_{g}\right)=$ $Q^{(1)}\left(a_{g}\right)+\frac{1}{2} s^{+}(g)$. This makes the metric homogenous of degree $d_{e}$ on all of $A$. Notice that $s^{+}(g)=s^{+}\left(g^{-1}\right)$.

The second alteration appears in step (2). For physically inspired reasons, one often makes an additional shift $\frac{1}{2} s^{-1}(g)$ depending on $g$ which has to preserve the homogeneity of the metric. The second shift satisfies $s^{-1}(g)=-s^{-1}\left(g^{-1}\right)$.

The final grading for an element $a_{g} \in A_{g}$ is

$$
\mathcal{Q}\left(a_{g}\right)=Q^{(2)}\left(a_{g}\right)+\frac{1}{2} s^{-}(g)=Q^{(1)}\left(a_{g}\right)+\frac{1}{2}\left(s^{+}(g)+s^{-}(g)\right) .
$$

If the $G$-action of step (2) is induced by a linear $G$ action there is a standard choice for this shift, given by 
Definition 1.7. The standard grading shift for a $G$-Frobenius algebra with a choice of linear representation $\rho: G \rightarrow G L_{n}(k)$ is given by

$$
s_{g}:=\frac{1}{2}\left(s_{g}^{+}+s_{g}^{-}\right)
$$

with

$$
s_{g}^{+}:=d-d_{g}
$$

and

$$
\begin{aligned}
s_{g}^{-}:=\frac{1}{2 \pi i} \operatorname{tr}(\log (g))-\operatorname{tr}\left(\log \left(g^{-1}\right)\right) & :=\frac{1}{2 \pi i}\left(\sum_{i} \lambda_{i}(g)-\sum_{i} \lambda_{i}\left(g^{-1}\right)\right) \\
& =\sum_{i: \lambda_{i} \neq 0}\left(\frac{1}{2 \pi i} 2 \lambda_{i}(g)-1\right)
\end{aligned}
$$

where the $\lambda_{i}(g)$ are the logarithms of the eigenvalues of $\rho(g)$ using the arguments in $[0,2 \pi)$.

This means that if $\rho(g)=\operatorname{diag}\left(\exp \left(2 \pi i \nu_{1}\right), \ldots, \exp \left(2 \pi i \nu_{n}\right)\right)$ with $0 \leq \nu_{i}<1$ then $\lambda_{i}=2 \pi i \nu_{i}$.

Remark 1.4. Notice that if $\rho(g)=\operatorname{diag}\left(\exp \left(2 \pi i \nu_{1}(g)\right), \ldots, \exp \left(2 \pi i \nu_{n}(g)\right)\right)$ with $0 \leq \nu(g)_{i}<1$ then

$$
\begin{gathered}
\nu_{i}\left(g^{-1}\right)=\left\{\begin{array}{ll}
0 & \text { if } \nu_{i}(g)=0 \\
1-\nu_{i}(g) & \text { else }
\end{array} .\right. \\
\nu_{i}(g h)=\nu_{i}(g)+\nu_{i}(h)-\Theta\left(1-\left(\nu_{i}(g)+\nu_{i}(h)\right)\right.
\end{gathered}
$$

where is the step function

$$
\Theta(x)= \begin{cases}1 & \text { if } x \geq 0 \\ 0 & \text { if } x<0\end{cases}
$$

Remark 1.5. In the case of orbifold cohomology $[\mathrm{CR}]$, one starts with an action of $G$ on the manifold $M$ and induces an action on the tangent space $M$ which defines the shift $s^{-}$via (1.5) and the shift $s_{g}^{+}$is defined by $d_{g}:=\operatorname{dim}(F i x(g) \subset M)$. For general orbifolds this reasoning is understood locally $[\mathrm{CR}]$. For global orbifolds the expressions can however be understood globally.

If $\rho(g)=\operatorname{diag}\left(\exp \left(2 \pi i \lambda_{1}\right), \ldots, \exp \left(2 \pi i \lambda_{n}\right)\right.$, then $d_{g}=\sum_{i: \lambda_{i}=0} 1$ and $d-d_{g}=$ $\sum_{i: \lambda_{i} \neq 0} 1$, so $s_{g}=\sum_{i: \lambda_{i} \neq 0} \frac{1}{2 \pi i} \lambda_{i}(g)=\sum_{i} q_{i}$ yielding agreement with the definition (1.3) above and the one of $[\mathrm{CR}]$ and $[\mathrm{Z}]$ in that particular case.

Notice that for the last expression of equation (1.5), we can use the branch of the logarithm obtained by cutting along $[0, \infty)$.

1.8.3. The super-grading. As for the grading, usually each $A_{g}$ comes with an intrinsic super-grading. In step (1) one usually allows the freedom to shift the super-grading by $\mathbb{Z} / 2 \mathbb{Z}$ values function. The restrictions on this function come from the existence of an even non-degenerate quasi-homogenous metric and in step (3) from the trace axiom. In step (4) the condition that $\chi$ is a character translates via the trace condition and the condition that $\chi^{2}$ is a character from step (2) into a condition on the super-grading. 


\subsubsection{Bi-grading.}

Definition 1.8. Set $\bar{s}_{g}:=\frac{1}{2}\left(s_{g}^{+}-s_{g}^{-}\right)$. Since $s_{g}^{+}=s_{g^{-1}}^{+}$and $s_{g}^{-}=-s_{g^{-1}}^{-}$if follows that $\bar{s}_{g}=s_{g^{-1}}$. We define that bi-grading $(\mathcal{Q}, \overline{\mathcal{Q}})$ by

$$
\mathcal{Q}\left(a_{g}\right):=Q\left(a_{g}\right)+s_{g} \quad \overline{\mathcal{Q}}\left(a_{g}\right):=Q\left(a_{g}\right)+\bar{s}_{g} \quad \text { for } a_{g} \in A_{g}=M_{\left.f\right|_{F i x}(g)}
$$

Remark 1.6. As mentioned in the introduction, the bi-grading has its origin in the interpretation for the algebra as the $(c, c)$ ring for an orbifold model [IV].

\section{A MIRROR TYPE TRANSFORMATION}

Assumptions: In this section for simplicity, we fix a $k=\mathbb{C}$. (If $k$ is a field of characteristic zero, we could fix an embedding $\bar{k} \hookrightarrow \mathbb{C}$.)

In the following, we will construct an involution for the triples $\langle A, j, \chi\rangle$ of $D(k[G])$-modules $A$, elements $j$ of the center of $G$ and characters $\chi \in \operatorname{Hom}\left(G, k^{*}\right)$.

In the case of an Euler $D(k[G])$ module, we take the element $j$ to be the element defined by the Euler property.

We also extend the operation to include a non-degenerate pairing and a bigrading.

This involution induces via restriction a dualization on quasi-Euler $D[k[G])$ modules (without pairing) with fixed Eulerization.

In the case $A$ is a Euler Frobenius algebra or a quasi-Euler Frobenius algebra with a fixed Eulerization, we let $Q$ be the grading operator and $j \in G$, s.t. $\rho(j)=$ $\exp (2 \pi Q)=J$. In this case the data $(A, j, \chi)$ is fixed by the $G$-Frobenius algebra and the element $j$ yielding the grading.

Our operation conjecturally acts as a mirror transformation on the underlying Euler $G$-Frobenius algebras in the sense of orbifold mirror philosophy, see $\S 2.6$.

The additional bi-grading, is conjecturally compatible with interchange of the $(c, c)$-type and $(a, c)$-type for Landau-Ginzburg theories w.r.t. mirror symmetry.

In fact, we will prove that the orbifold mirror philosophy is correct in the case of the simple singularities $A_{n}, D_{n}, E_{6}, E_{7}, E_{8}$ and yields mirror pairs for the simple boundary singularities $B_{n}, F_{4}$.

Remark 2.1. The definition of the dual comes from physics [IV, V], where the dual $D(k[G])$-module is obtained by using an endofunctor in the category of representations of the $N=2$ super-conformal algebra which translates in our case to an isomorphism of $D(k[G])$-modules. This endofunctor is generally known as spectral flow and has a particular realization discussed below in the case of $G$-Frobenius algebras.

\subsection{The $G$-graded $k$-module structure.}

Definition 2.1. Given a $G$-graded $k$-module $A$ and an element $j \in Z(G)$ we define the dual $\check{A}$ to be the $G$-graded $k$ module:

$$
\check{A}_{g}:=A_{g j^{-1}}, \quad \check{A}:=\bigoplus_{g \in G} \check{A}_{g}
$$

Remark 2.2. The above formula states that as $k$ modules $A$ and $\check{A}$ are isomorphic. It is only their $G$-grading which has changed. We denote the isomorphism by $M: A \rightarrow \check{A}$, with $M\left(A_{g}\right)=\check{A}_{g j}$. 
2.2. The metric. With the help of the map $M^{-1}$, we can pull back a given metric $\eta$ from $A$ to $\check{A}$. We set

$$
\check{\eta}=\left(M^{-1}\right)^{*} \eta \quad \check{\eta}(\check{a}, \check{b}):=\eta\left(M^{-1}(\check{a}), M^{-1}(\check{b})\right)
$$

Remark 2.3. Notice if $\eta$ is homogeneous with respect to the group degree, i.e. pairs $A_{g}$ with $A_{g^{-1}}$, then $\check{\eta}$ pairs $\check{A}_{g}$ with $\check{A}_{g^{-1} j^{2}}$ and thus $\check{\eta}$ is not group degree homogeneous, but of group degree $j^{2}$ as a tensor in $\check{A}^{*} \otimes \check{A}^{*}$.

Remark 2.4. The metric $\check{\eta}$ is $G$-invariant and hence descends to the $G$-invariants.

2.3. The $G$-action or the $D(G[k])$-module structure. Given a triple $\langle A, j, \chi\rangle$ of a $D(k[G])$-module $A$, an element $j$ of the center of $G$ and a character $\chi \in$ $\operatorname{Hom}\left(G, k^{*}\right)$, we define $\bar{\varphi}:=\varphi \otimes_{k} \chi$. This is an action of $G$ on the $k$-module $A \otimes_{k} k \simeq A$ and thus on the $k$-module $\check{A}$.

We define the $G$-action $\check{\varphi}$ on $\check{A}$ to be the induced action of the action on $A$ by $\bar{\varphi}$. That is for $a \in \check{A}$

$$
\check{\varphi}(h)(a)=\chi(h) M\left(\varphi(h)\left(M^{-1}(a)\right)\right)
$$

Remark 2.5. We see that under this action $\check{\varphi}(h)\left(\check{A}_{g}\right) \subset \check{A}_{h g j^{-1} h^{-1} j}=A_{h g h^{-1}}$, since we made the assumption that $j \in Z(G)$. Thus we obtain a $G$-action, which makes $\check{A}$ into a $D(k[G]])$-module.

2.4. The bi-grading of the dual. If $A$ was initially graded by the operator $Q^{(1)}$ and or simply $Q\left(a_{g}\right)=Q^{(1)}\left(a_{g}\right)+s_{g}$ then.

Set $\check{s}_{g}:=s_{g j^{-1}}-d$ and $\check{s}_{g}:=\bar{s}_{g j^{-1}}$, where we recall that $d$ is the degree of the $G$-Frobenius algebra. We define a bi-grading on $\check{A}$ by

$$
\check{\mathcal{Q}}(\check{a})=Q^{(1)}(a)+\check{s}_{g} \quad \overline{\mathcal{Q}}:=Q^{(1)}(a)+\bar{s}_{g} \quad \text { for } \check{a}_{g} \in \check{A}_{g}
$$

Remark 2.6. For an Euler $G$-Frobenius algebra $A=<G, A, \circ, 1, \eta, \varphi, \chi, j>$ naturally gives rise to a triple $\langle A, j, \chi>$ and thus obtain a dual $D(k[G])$ module with a non-degenerate pairing and a bi-grading.

Remark 2.7. The motivation for the dual bi-grading again comes from the physical interpretation of $G M_{f}$ as an orbifold Landau-Ginzburg model and the dualization being implemented by the spectral flow operator $\mathcal{U}_{(1,0)}[\mathrm{IV}]$ which has the natural charge $\left(d=\hat{c}=\frac{c}{3}, 0\right)$.

\subsubsection{The involution.}

Definition 2.2. We define the dual of a triple $\langle A, j, \chi\rangle$ of $D(k[G])-$ modules $A$, elements $j$ of the center of $G$ and characters $\chi \in \operatorname{Hom}\left(G, k^{*}\right)$ to be the triple $\left\langle\check{A}, j^{-1}, \chi^{-1}\right\rangle$.

Remark 2.8. Notice that the inclusion of the data $j$ and $\chi$ turns the operation on the $D(k[G])$-module into an involution.

2.4.2. The dual of a quasi-Euler $D[k[G])$-module with given Eulerization.

Definition 2.3. We define the dual of a quasi-Euler $D[k[G]$ )-module $A$ (or $H$ Frobenius algebra) with given Eulerization $B$ to be the restriction of $\check{B}$ to $H$. $\check{A}:=$ $\operatorname{res}_{H}(\check{B})$ 
Remark 2.9. Notice that if $j \notin H$ then we cannot pull back the metric, since if $h \in H, h^{-1} j^{2}$ need not be in $H$. If $\forall h \in H: h^{-1} j^{2} \in H$, then we can also pull back the metric.

2.5. A dual $G$-Frobenius algebra? We would like to remark that the dualizing process is only a process of dualizing for $D(k[g])$ modules with metric.

One thing to prevent the resulting structure from being a $G$-Frobenius algebra is that the metric is not $G$-graded anymore as remarked above in Remark 2.3. Also the projective self-invariance might not hold. However, there might be, in some cases unique, choices of $G$-graded multiplication compatible with the $G$-action.

Or what is actually expected by physics, that there is a Frobenius algebra structure on the $G$-invariants of $\check{A}$ with the given metric. It is important to note that physics does not say there should be an algebra isomorphism and in fact the induced multiplication $M \circ M^{-1}$ will not be $G$-graded on $\check{A}$ unless $j=e$ and the grading and dualization are trivial.

What we can expect is a Frobenius structure on the invariants, plus a lift of this Frobenius structure to the $G$-graded equivariant level. This will provide some additional structure. This motivates the following definition.

Definition 2.4. A degenerate $G$-Frobenius algebra $A$ of degree $j \in Z(G)$ is given by the data $\left\langle G, A, \circ, 1, \eta, \eta^{\prime}, \varphi, \chi\right\rangle$ where $\langle G, A, \circ, 1, \eta, \varphi, \chi>$ are the data of a $G-$ Frobenius algebra, and $\eta^{\prime}$ is a second pairing on $G$. These data satisfy the conditions of a $G$-Frobenius algebra with the following changes and additions:

1) The non-degenerate paring $\eta$ and the pairing $\eta^{\prime}$ pair $A_{g j^{-1}}$ with $A_{g^{-1} j^{-1}}$.

2) $\left.\eta\right|_{A^{G}}=\eta_{A^{G}}^{\prime}$ where $A^{G}$ are the $G$ invariants of $A$.

d') Invariance of the metric $\eta^{\prime}$ :

$$
\eta^{\prime}\left(a_{g}, a_{h} \circ a_{k}\right)=\eta^{\prime}\left(a_{g} \circ a_{h}, a_{k}\right)
$$

i) ${ }^{j}$ Self-invariance of the twisted sectors

$$
\varphi_{g j^{-1}} \mid A_{g}=i d
$$

iii $\left.^{j}\right) G$-invariance of the metric $\eta$

$$
\varphi_{g}^{*}(\eta)=\eta
$$

iv $\left.^{j}\right) j$ twisted trace axiom $\forall c \in A_{[g, h]}$ and $l_{c}$ left multiplication by $c:$ $\operatorname{Tr}\left(\left.l_{c} \varphi_{h j^{-1}}\right|_{A_{g}}\right)=\operatorname{Tr}\left(\left.\varphi_{g^{-1} j} l_{c}\right|_{A_{h}}\right)$

Conjecture 2.1. We conjecture that there is a degenerate $G$-Frobenius algebra of degree $j$ on the dual of a $G$-Euler $G$-Frobenius algebra.

In the examples we consider, there is a certain uniqueness in the choice for the multiplication. In order to state this precisely we need the following two definitions.

Definition 2.5. Fix a $D(k[G]), A_{e}$ bi-module $A=\bigoplus A_{g}$ together with two metrics $\eta, \eta^{\prime}$ and a character $\chi$ satisfying all the axioms pertaining to the metrics and the $G$-actions of a degenerate $G$-Frobenius algebra of degree $j$. We call a degenerate $G$-Frobenius algebra $A$ of degree $j$ maximally non-degenerate if $a_{g} \circ b_{h}=0$ in $A$ implies that $a_{g} \circ^{\prime} b_{h}=0$ in any other degenerate $G$-Frobenius algebra $A^{\prime}$ with the same underlying $D(k[G]), A_{e}$ bi-module $A^{\prime}=\bigoplus A_{g}$ together with the two metrics $\eta, \eta^{\prime}$ and the character $\chi$.

We call a maximally non-degenerate $G$-Frobenius algebra projectively unique if it agrees with all other maximally non-degenerate $G$-Frobenius structures when projected to $A / k^{*}$. 
Remark 2.10. As demonstrated in [Ka6] twisting by discrete torsion exactly realizes the universal (i.e. applicable to all $G$-Frobenius algebras) projective rescalings. This means vice versa that $A$ and all its twists by discrete torsion are projectively the same.

2.6. Orbifold mirror philosophy. There is a orbifold mirror philosophy which is motivated by physics (cf. eg. [GP]) or representation theory which states the following

Philosophy 2.1. Let $T$ be a $N=2$ theory (which for us at the moment means Frobenius algebra) and let $H \subset G$ be symmetry groups with $H$ normal in $G$ then

$$
(T / H)^{H} \simeq\left(((T / H) /(G / H))^{\vee}\right)^{(G / H)}
$$

for us $T / K$ means a $K$-Frobenius algebra derived from $K$.

This is too vague to be called a conjecture, since most of the symbols in the statement have no fixed meaning. We can however apply it to quasi-homogenous singularities, where up to a finite amount of data $(\sigma, \epsilon, \gamma)$ if $G$ is Abelian see $\S 3$ below) the ingredients $T / K$ are fixed.

It turns out that even for different actions of $H$ and $G$ this orbifold mirror philosophy holds true.

In order to elucidate the statement, we wish to point out that there is indeed an action of $G / H$ on the $H$ invariants of a $G$-Frobenius algebra $A$. For the action to be defined on the restriction $\operatorname{res}_{H}(A)$ we need the addition assumption that $H$ is normal. Therefore the statement makes sense on the level of $D(k[G])$-modules once the $G$-action is fixed.

\section{QuASI-HOMOGENOUS SINGULARITIES WITH SYMMETRIES}

We again fix $k=\mathbb{C}$.

Definition 3.1. Let $f: \mathbb{C}^{n} \rightarrow \mathbb{C}$ be a function which has an isolated singularity at zero. A symmetry of $f$ is an element $S \in G L(n, \mathbf{C})$, s.t. $f(S(\mathbf{z}))=f(\mathbf{z})$. An isolated singularity with symmetries is a function $f: \mathbb{C}^{n} \rightarrow \mathbb{C}$ which has an isolated singularity at zero together with a finite group $G$ and a representation $\rho(G)$ : $G \rightarrow G L(n, \mathbb{C})$ such that $G$ acts by symmetries on $f$, i.e. $\forall g \in G: g^{*}(f)(\mathbf{z})=$ $f(\rho(g)(\mathbf{z}))=f(\mathbf{z})$.

We denote by $G_{\max } \subset G L(n, \mathbb{C})$ the maximal group of symmetries of $f$.

Definition-Proposition 3.1. For a function $f(\mathbf{z})$ with an isolated singularity at zero. We will denote by $M_{f}$ the Milnor or local ring of $f$, which is given by $\mathcal{O} / J_{f}$ where $\mathcal{O}$ is the ring of germs of holomorphic functions at zero and $J_{f}=\left(\frac{\partial f}{\partial z_{i}}\right)$ is the Jacobian ideal. This ring together with the Grothendieck residue paring $\eta$ is a graded Frobenius algebra, see e.g. [AGLV, M].

3.1. The graded Frobenius algebra of a quasi-homogenous with an isolated singularity at zero. If the function $f$ is also quasi-homogenous there is a natural grading operator which assigns to each $z_{i}$ its degree of quasi-homogeneity $q_{i}$.

To define the $q_{i}$ assume that

$$
f\left(\lambda^{Q_{1}} z_{1}, \ldots, \lambda^{Q_{n}} z_{n}\right)=\lambda^{N} f\left(z_{1}, \ldots, z_{n}\right)
$$


with $Q_{i}, N \in \mathbb{N}$. Then we set $q_{i}=\frac{Q_{i}}{N}$ and define $\operatorname{deg}\left(z_{i}\right):=q_{i}$ which yields a map $M_{f} \rightarrow \mathbb{Q}$.

The metric for the resulting Frobenius algebra is given by the element which is dual to the identity and this element is represented by $H:=H e s s(f)$ the Hessian of $f$. For a quasi-homogenous singularity, the degree of the Hessian is the degree of the form $\eta$ and is denoted by $d$. By the general theory [AGLV] a formula for $d$ is given by

$$
d=\sum_{i}\left(1-2 q_{i}\right)
$$

and the dimension or the Milnor number of the local algebra is

$$
\mu:=\operatorname{dim}\left(M_{f}\right)=\prod_{i}\left(\frac{1}{q_{i}}-1\right)
$$

3.1.1. Examples. In the following examples, we took the liberty to re-scale the Grothendieck residue form, which amounts to adding an overall factor to the function $f$.

(1) The $A_{n}$ series: $f(z, w)=z^{n+1}$

$$
\begin{aligned}
& M_{f}=\mathbb{C}[z] /\left(z^{n-1}\right)=\left\langle 1, z, z^{2}, \ldots, z^{n-1}\right\rangle \quad \eta\left(z^{i}, z^{j}\right)=\delta_{i+j, n-1} \\
& q=\frac{1}{n+1}, \mu=n, d=1-\frac{2}{n+1}=\frac{n-1}{n+1} .
\end{aligned}
$$

(2) The $D_{n}$ series: The $D_{n+1}, n \geq 3$ singularity is given by the function $f(x, y, w)=\frac{1}{n} x^{n}+x y^{2} M_{f} \simeq \mathbb{C}[x, y] /\left(x^{n}, x y\right) \simeq\left\langle 1, x, x^{2}, \ldots, x^{n-2}, y\right\rangle$ $\eta\left(x^{i}, x^{j}\right)=\delta_{i+j, n-1}, \eta(y, y)=1, \eta\left(x^{i}, y\right)=0$ $q_{x}=q_{1}=\frac{1}{n}, q_{y}=q_{2}=\frac{n-1}{2 n}, \mu=n, d=\frac{n-1}{n}$

(3) The $E_{7}$ singularity: $\frac{1}{3} x^{3}+x y^{3}$

$$
\begin{aligned}
& M_{f}=\mathbb{C}[x, y] /\left(x^{3}, x y^{2}\right)=\left\langle 1, x, x^{2}, y, y^{2}, x y, x^{2} y, x^{n-2}, y\right\rangle \\
& \eta\left(x^{i} y^{j}, x^{k} y_{l}\right)=\delta_{i+k, 2} \delta_{j+l, 1} \\
& q_{x}=q_{1}=\frac{1}{3} q_{y}=q_{2}=\frac{2}{9}, \mu=6, d=\frac{8}{9} .
\end{aligned}
$$

3.1.2. Products. For two functions $f$ and $g$ with an isolated singularity at zero, as shown in [Ka1, M $] M_{f+g}=M_{f} \otimes M_{g}$ even on the level of Frobenius manifolds.

3.1.3. Stabilization. Notice that adding squares, an operations known as stabilization, to a function with an isolated singularity $\left(f \mapsto f+w^{2}\right)$ leaves the Milnor ring invariant. This fact which is well known in singularity theory (see e.g. [AGLV]), can also be seen as follows from the point of view of Frobenius algebras.

Since the Frobenius algebra of the singularity $f(w)=w^{2} M_{w^{2}}=A_{1}=k$ is the unit in the monoidal category of Frobenius algebras [Ka1] we also find that $M_{f+w^{2}} \simeq M_{f} \otimes A_{1} \simeq M_{f}$

In the following, all the definitions, calculations and operations are invariant under stabilization.

Definition 3.2. We define $M_{0}:=M_{w^{2}}=A^{1}$.

Remark 3.1. All the following definitions and constructions are invariant under stabilizations, if one extends the group action by the usual embedding of $G L(n, \mathbb{C})$ to $G L(n+1, \mathbb{C})$. 
3.2. The $G$-Frobenius algebra for a singularity with symmetry group $G$. We would like to recall from [Ka2, Ka3, Ka4, Ka5] that for the data $(f, G, \rho)$ as above there are several natural $G$-Frobenius algebras, whose underlying $k$-module structure and bi-grading are all the same, but whose $D(k[G])$ module structures are in one - to - one correspondence with twists by discrete torsion and whose $G^{-}$ Frobenius structures depend on the choice of a graded compatible co-cycle for the quantum multiplication. We will review the construction below following the steps of $\S 1.7$.

3.2.1. The $G$-graded $k$-module structure. First we show that for the data $(f, G, \rho)$ as above there is a natural associated $G$-graded $M_{f}$ module.

Let $\operatorname{Fix}_{g}:=$ the fixed point set of $g$ in $\mathbb{C}^{n}$, in other words the eigenspace to the eigenvalue 1 of $\rho(g)$. Set $f_{g}:=\left.f\right|_{\mathrm{Fix}_{g}}$.

We define

$$
A_{g}:=M_{f_{g}} \quad G M_{f}:=\bigoplus_{g \in G} M_{f_{g}}
$$

Remark 3.2. We would like to emphasize the following observations:

1) Notice $A_{e}=M_{f}$.

2) Each of the $A_{g}=M_{f_{g}}$ is as a local ring of a quasi-homogenous function with an isolated singularity at zero is a Frobenius algebra. We denote the metric for the Frobenius algebra $A_{g}$ by $\eta_{g}$, its unit by $1_{g}$ and its degree by $d_{g}$. and its grading operator by $Q_{g}$. A.

These sum of the grading operators $Q_{g}$ defines a grading operator $Q$ on

3) We furthermore can use the ring structure of the individual $M_{f_{g}}$ to define natural $M_{f}$ module structure by inclusion of function germs. This $A_{e^{-}}$ module structure is compatible with the grading by $Q$.

In the following examples the multiplication is given by ${ }^{1}$ :

$$
M_{f} \times M_{f_{g}} \rightarrow M_{f_{g}}:\left.(a, b) \mapsto a\right|_{\text {Fix }_{g}} b
$$

Remark 3.3. All the $A_{g}$ are cyclic $A_{e}$ modules. In the terminology of [Ka3] $G M_{f}$ is a special $G$-Frobenius algebra. Notice that the unit $1_{g}$ is a cyclic generator for the $A_{e}$ module $A_{g}$.

3.2.2. The grading. The initial grading operator $Q$ from above plays the role of the operator $Q^{(1)}$ of $\S 1.8$. The actual grading $Q$ is determined by the degrees of the cyclic generators $1_{g}$.

Definition 3.3. We define the grading operator $\mathcal{Q}$ on $G M_{f}$ by

$$
\mathcal{Q}\left(a_{g}\right)=Q(a)+s_{g} \text { for } a_{g}=a 1_{g}
$$

with

$$
s_{g}=\frac{1}{2}\left(s_{g}^{+}+s_{g}^{-}\right)=\frac{1}{2}\left(d-d_{g}\right)+\sum_{i: \nu_{i} \neq 0}\left(\frac{1}{2 \pi i} \lambda_{i}(g)-\frac{1}{2}\right)
$$


$z_{i}: i \in I_{1}$ be a basis of Fix $(g)$ and $z_{i}: i \in I_{2}$ a basis of the complement of the fixed point set. Set $Z=\left(z_{i}: i \in I_{2}\right)$ let $J_{g}=J_{f_{g}}$ and $J_{I_{1}}=\left(f_{z_{i}}: i \in I_{1}\right)$ be the respective ideals in $\mathcal{O}$. Then the condition is that $J_{I_{1}}+Z=J_{g}+Z$. In the other case the map still exists by functoriality, but is a little more complicated.
} 
where the $\lambda_{i}(g)$ are the logarithms of the eigenvalues of $g$ using the branch with arguments in $[0,2 \pi)$ i.e. cut along the positive real axis.

This means that

$$
s_{g}=\mathcal{Q}\left(1_{g}\right)
$$

and we call $s_{g}$ the grading shift.

3.2.3. Notation. In practice the choice of logarithms means that in a diagonal form $\rho(g)=\operatorname{diag}\left(\exp \left(2 \pi i \nu_{1}(g)\right), \ldots, \exp \left(2 \pi i \nu_{n}(g)\right)\right.$ and $0 \leq \nu_{i} \leq 1$.

For the element $j, \nu_{i}(j)=q_{i}$, also due to choice of logarithm $\nu_{i}(g)=1-\nu_{i}\left(g^{-1}\right)$ furthermore $d_{g}=\sum_{i: \nu_{i}(g)=0} 1-2 q_{i}$ and so

$$
\begin{aligned}
s_{g}^{+} & =2 \sum_{i: \nu_{i}(g) \neq 0}\left(\frac{1}{2}-q_{i}\right), \quad s_{g}^{-}=2 \sum_{i: \nu_{i}(g) \neq 0}\left(\nu_{i}(g)-\frac{1}{2}\right) \\
s_{g} & =\sum_{i: \nu_{i}(g) \neq 0}\left(\nu_{i}(g)-q_{i}\right) .
\end{aligned}
$$

3.2.4. The bi-grading. There is a natural bi-grading for the $G$-Frobenius algebras of the type $G M_{f}$ which is given by

$$
(\mathcal{Q}, \bar{Q})\left(a_{g}\right):=\left(Q\left(a_{g}\right)+s_{g}, Q\left(a_{g}\right)+\bar{s}_{g}\right) \quad \text { for } a_{g} \in A_{g}
$$

where we used the notation $\bar{s}_{g}:=\frac{1}{2}\left(s_{g}^{+}-s_{g}^{-}\right)$.

In the notation above

(3.3) $\bar{s}_{g}=\sum_{i: \nu_{i}(g) \neq 0}\left(1-\nu_{i}(g)-q_{i}\right)=\sum_{i: \nu_{i}\left(g^{-1}\right) \neq 0}\left(\nu_{i}\left(g^{-1}\right)-q_{i}\right)=s_{g^{-1}}=d-d_{g}-s_{g}$

Remark 3.4. This grading is physically motivated, [IV], and basically means that the natural bi-degree of the so-called "twist field" is $\left(s_{g}, \bar{s}_{g}\right)$.

Lemma 3.1. An element $a_{g} \in A_{g}$ has diagonal grading $(q, q)$ if and only if $s_{g}=$ $s_{g^{-1}}$, i.e. $s_{g}^{-}=0$ or equivalently $\sum_{i} \nu_{i}=\frac{1}{2} \operatorname{codim}(\operatorname{Fix}(\mathrm{g}))$.

3.2.5. Super-grading. Recall that $A_{g}$ are all cyclic $A_{e}$ modules and the natural parity for all of $A_{e}$ is all even. Thus under the assumption that all elements of $A_{e}$ are even the possible super-gradings for the $M_{f}$ module $G M_{f}$ are given by maps $\sim \in \operatorname{Map}(G, \mathbb{Z} / 2 \mathbb{Z})$. Here $\tilde{1}_{g}=\tilde{g}$. Here we use $\sim$ both for the grading on $A$ and $G$ which is justified by the equation above.

3.2.6. The $G$-actions. The primary choice for a $G$-action on $G M_{f}$ would be the induced action via pullback. Since $G$ acts on the collection of fixed point sets: $h: \operatorname{Fix}_{g} \rightarrow$ Fix $_{h g h^{-1}}$, we get a right action $r$ on $G M_{f}$ which coincides with the notation of $[\mathrm{JKK}]$. On the other hand, if we take the associated left action $l$, we are in line with the definitions of [Ka3] - here $l(g):=r\left(g^{-1}\right)$.

However, at this stage all actions of $G$ are good which preserve the structures. Now each $A_{g}$ is a cyclic $A_{e}$ module and we denote the generator by $1_{g}$. We find that if $G$ is acting via $A_{e}$ module automorphisms then

$$
\varphi(g) 1_{h}=\varphi_{g, h} 1_{g h g^{-1}} \text { for some } \varphi_{g, h} \in k^{*}
$$

From the fact that this is indeed an action of $G$, we obtain a co-cycle condition on the $\varphi_{g, h}$. To be precise, they form a non-abelian $G 2-$ co-cycle with values in $k^{*}$, where: 
Definition 3.4. A non-abelian $G$ 2-cocycle with values in $k^{*}$ is a map $\varphi: G \times G \rightarrow$ $K^{*}$ which satisfies:

$$
\varphi_{g h, k}=\varphi_{g, h k h^{-1}} \varphi_{h, k}
$$

where $\varphi_{g, h}:=\varphi(g, h)$ and

$$
\varphi_{e, g}=\varphi_{g, e}=1
$$

3.2.7. The super-grading. To define the super-grading we make an additional assumption.

Assumption Keeping the condition that all elements of $A_{e}$ are even, we furthermore postulate that the $G$-action is an even action.

This limits the possible super-gradings to functions of $C(G) \rightarrow \mathbb{Z} / 2 \mathbb{Z}$ where $C(G)$ are the conjugacy classes of $G$.

3.2.8. The conditions from the trace axiom. As demonstrated in [Ka3], if we further demand that the restricted trace axiom holds ${ }^{2}$ for the above $D(k[G])$ module, certain conditions for the character, the super-grading and the co-cycle $\varphi_{g, h}$ must hold.

Recall $\rho: G \rightarrow G L(n, \mathbf{C})$ is the representation fixed from the beginning.

3.2.9. The trace axiom, the character and the super-grading. From the proof of the Theorem 5.1. [Ka3] we extract the following conditions proposition:

Proposition 3.2. Let $G M_{f}$ be the $D(k[G])$-module with the $G$-action given by the co-cycle $\varphi_{g, h}$ and fix a super-grading $\sim$ and a character $\chi \in \operatorname{Hom}\left(G, k^{*}\right)$, then the trace axiom for $g=e$ and arbitrary $h$ is satisfied w.r.t. $\sim$ and $\chi$ if and only if $\chi$ satisfies

$$
\chi_{g}=(-1)^{\tilde{g}}(-1)^{n-\operatorname{dim}\left(\operatorname{Fix}_{g}\right)} \operatorname{det}(\rho(g))
$$

Remark 3.5. Notice that this entails a condition on the super-grading:

Set

$$
\sigma(g):=\tilde{g}+n-\operatorname{dim}(\operatorname{Fix}(g)) \bmod 2
$$

and call it the sign of $g$. Then

$$
\chi_{g}=(-1)^{\sigma(g)} \operatorname{det}(g)
$$

and therefore $\sigma \in \operatorname{Hom}(G, \mathbb{Z} / 2 \mathbb{Z})$.

Thus the possible super-gradings $\sim$ are in 1-1 correspondence with elements $\sigma$ of $\operatorname{Hom}(G, \mathbb{Z} / 2 \mathbb{Z})$.

\subsubsection{The trace axiom and discrete torsion.}

Definition 3.5. A discrete torsion bi-character for a group $G$ is a map from commuting pairs $(g, h) \in G \times G:[g, h]=e$ to $K^{*}$ with the properties:

$$
\epsilon(g, h)=\epsilon\left(h^{-1}, g\right) \quad \epsilon(g, g)=1 \quad \epsilon\left(g_{1} g_{2}, h\right)=\epsilon\left(g_{1}, h\right) \epsilon\left(g_{2}, h\right)
$$

Definition 3.6. A non-abelian 2-cocycle is said to satisfy the condition of discrete torsion with respect to a given $\sigma \in \operatorname{Hom}(G, \mathbb{Z} / 2 \mathbb{Z})$ and a linear representation $\rho \in \operatorname{Hom}(G, G L(n))$, if for all elements $g, h \in G:[g, h]=e$ :

$$
\epsilon(g, h):=\varphi_{g, h}(-1)^{\sigma(g) \sigma(h)} \operatorname{det}(g) \operatorname{det}\left(\left.g^{-1}\right|_{\operatorname{Fix}(h)}\right)
$$

is a discrete torsion.

\footnotetext{
${ }^{2}$ This means $[g, h]=e, c=1$
} 
Remark 3.6. Due to the properties of $\varphi$ as a non-abelian cocycle, $\varphi$ the second and third condition of discrete torsion (3.6) are automatically satisfied. If furthermore $\gamma_{g, h} \neq 0$ then the first condition reduces to

$$
\operatorname{det}(g) \operatorname{det}\left(\left.g^{-1}\right|_{\operatorname{Fix}(h)}\right) \operatorname{det}(h) \operatorname{det}\left(\left.h^{-1}\right|_{\operatorname{Fix}(g)}\right)=1 .
$$

3.2.11. The action of discrete torsion. In [Ka6], we analyzed the phenomenon of discrete torsion and showed that the different choices $\varphi$ can by obtained from a fixed $D(k[G])$ module by tensoring with the twisted group algebra $k^{\alpha}[G]$ with $\alpha \in Z^{2}\left(G, k^{*}\right)$.

The corresponding discrete torsion bi-character to such an $\alpha \in Z^{2}\left(G, k^{*}\right)$ is given by

$[\mathrm{Ka} 6]$

$$
\epsilon(g, h)=\frac{\alpha(g, h)}{\alpha\left(g h g^{-1}, g\right)}
$$

From the considerations of [Ka6] one obtains:

Lemma 3.2. For two choices of non-abelian cocycles $\varphi$ and $\varphi^{\prime}$, let $A(\varphi)$ and $A\left(\varphi^{\prime}\right)$ be the $D(k[G])$ modules based on the $k$-module $G M_{f}$, then there is a group cocycle $\alpha \in Z^{2}\left(G, k^{*}\right)$, s.t. that $A\left(\varphi^{\prime}\right) \simeq A(\varphi) \otimes k^{\alpha}[G]$.

From the Proof of Theorem 5.1. of [Ka3] we can also extract the following:

Proposition 3.3. The $D(k[G])$-module $G M_{f}$ with the $G$-action given by the cocycle $\varphi_{g, h}$ satisfies the super-trace axiom if and only if there is a $\sigma \in \operatorname{Hom}(G, \mathbb{Z} / 2 \mathbb{Z})$ s.t. $\varphi_{g, h}$ satisfies the condition of discrete torsion with respect to a $\sigma \in \operatorname{Hom}(G, \mathbb{Z} / 2 \mathbb{Z})$ and the linear representation $\rho \in \operatorname{Hom}(G, G L(n))$.

Corollary 3.1. If the group $G$ is Abelian then specifying a $G$ action by a nonabelian cocycle $\varphi$ which satisfies the restricted trace axiom for the resulting $D(k[G])$ module is equivalent to specifying a discrete torsion bi-character $\epsilon(g, h)$ and a group homomorphism $\sigma \in \operatorname{Hom}(G, \mathbb{Z} / 2 \mathbb{Z})$

$$
\varphi_{g, h}=\left.\epsilon(g, h)(-1)^{\sigma(g) \sigma(h)} \operatorname{det}\left(g^{-1}\right) \operatorname{det}(g)\right|_{\operatorname{Fix}(h)}
$$

3.2.12. The $G$-Frobenius algebra structures. As explained in [Ka3] and [Ka4] there is no fixed preferred $G$ Frobenius structure on the $M_{f}$ module above in general, but rather a set depending on the choice of a so-called super-sign and a two cocycle. The main result of [Ka3] in this respect is:

Theorem 3.1 ([Ka3]). Given a natural $G$ action on a realization of a Jacobian Frobenius algebra $\left(A_{e}, f\right)$ with a quasi-homogeneous function $f$ let $A:=\bigoplus_{g \in G} A_{g}$ be as defined above up to an isomorphism of Frobenius algebras on the $A_{g}$ then the structures of super $G$-Frobenius algebra on $A$ are in 1-1 correspondence with triples $(\sigma, \gamma, \varphi)$ where $\sigma \in \operatorname{Hom}(G, \mathbb{Z} / 2 \mathbb{Z}), \gamma$ is a $G$-graded, section independent cocycle compatible with the metric satisfying the condition of supergrading with respect to the natural $G$ action, and $\varphi$ is a non-abelian two cocycle with values in $K^{*}$ which satisfies the condition of discrete torsion with respect to $\sigma$ and the natural $G$ action, such that $(\gamma, \varphi)$ is a compatible pair.

In many cases the equations for the co-cycles allow one to find a unique multiplication up to the twist by discrete torsion.

We refer the reader to [Ka3] for details. 
The co-cycle $\gamma$ is a special type of $A_{e}$ valued group 2-cocycle which defines on the cyclic generators multiplication via

$$
1_{g} \circ 1_{h}:=\gamma(g, h) 1_{g h}
$$

the extra conditions ensure that the extension of this multiplication using the cyclic $A_{e}-$ module structures is well defined. We usually write $\gamma_{g, h}$ for $\gamma(g, h)$

The function $\sigma$ is related to the super-sign as follows.

$$
\sigma(g):=\tilde{g}+\left|N_{g}\right| \bmod 2
$$

where $\left|N_{g}\right|:=\operatorname{codim}($ Fix $(g))$ in $\mathbb{C}^{n}$.

Also:

Definition 3.7. A cocycle $\gamma \in Z^{2}\left(G, A_{e}\right)$ is said to satisfy the condition of supergrading with respect to a given a linear representation $\rho \in \operatorname{Hom}(G, G L(n))$, if $\gamma_{g, h}=0$ unless $\left|N_{h}\right|+\left|N_{g}\right|+\left|N_{g h}\right| \equiv 0(2)$. Here $\left|N_{g}\right|:=\operatorname{codim}($ Fix $(\rho(g))$ is the codimension of the fixed point set of $g$.

3.2.13. The metric. The metric is constructed in two steps.

In the first step the metric is constructed from the metrics on the individual Frobenius algebras $A_{g}:=M_{f_{g}}$. First notice that since $\operatorname{Fix}(g)=\operatorname{Fix}\left(g^{-1}\right): A_{g}=$ $A_{g^{-1}}$. Now $A_{g}$ has a non-degenerate pairing $\eta_{g}$ which we wish to view as a pairing $A_{g} \otimes A_{g^{-1}} \rightarrow k$. We set

$$
\eta^{\prime}:=\bigoplus_{g \in G} \eta_{g} \in A^{*} \otimes A^{*}
$$

In order to ensure the compatibility of the metric with the multiplication and the twisted commutativity $\gamma_{g, g^{-1}}=(-1)^{\tilde{g}} \varphi_{g, g^{-1}} \gamma_{g^{-1}, g}$ we need to rescale the metric:

$$
\eta:=\bigoplus_{g \in G}\left((-1)^{\tilde{g}} \chi_{g}\right)^{1 / 2} \eta_{g}
$$

For a discussion of the choice of the square root we refer to [Ka3].

3.3. The metric on the invariants. As shown in [Ka3] the $G$-invariants will be a Frobenius algebra with respect to the metric $\eta$ if and only if $\chi(g)= \pm 1$.

Remark 3.7. In physics terms, this means that the spectral flow operator $\mathcal{U}_{(1,1)}$ survives in the projection.

\subsection{The dual of a quasi-Euler $G$-Frobenius algebra for a quasi-homogenous singularity with symmetries.}

3.4.1. The grading operator and the Euler condition. Any non-trivial Frobenius algebra $M_{f}$ stemming from a quasi-homogenous singularity has a nontrivial grading operator $Q$ as discussed above and

$$
J:=\exp (2 \pi i Q)=\operatorname{diag}\left(\exp \left(2 \pi i q_{1}\right), \ldots, \exp \left(2 \pi i q_{n}\right)\right)
$$

generates a non-trivial finite cyclic group $\langle J\rangle \subset G L(n)$ of order $\operatorname{ord}(J)$ the order of $J$. Moreover fixing $J$ as the generator we can identify this group with $\mathbb{Z} / \operatorname{ord}(J) \mathbb{Z}$ with a fixed generator $j$ acting via $\rho(j)=J$.

Remark 3.8. This means that $G_{\max }$ is non-trivial. Since any symmetry has to preserve the grading $j$ it is in the center of $G_{\max }$ and thus any of the $G_{\max }$ Frobenius algebras constructed in $\$ 3.2$ will be Euler. This will also be true for any subgroup $H \subset G_{\max }$ which contains $\langle J\rangle$. 
Lemma 3.3. If $\forall g: \epsilon\left(g, j^{-1}\right)(-1)^{\sigma(g)(\sigma(j)+1)}=1$ then the corresponding $G-$ Frobenius algebras of $\S 3.2$ will be $G-E u l e r$. This is for instance the case if $\forall \epsilon(g, j) \equiv$ 1 and $\sigma(j)=1$ or $\sigma \equiv 0$

Proof.

$$
\varphi_{h^{-1} j, h}=\epsilon(h j, h)(-1)^{\sigma\left(h^{-1} j\right) \sigma(h)} \exp \left(2 \pi i \sum_{i: \nu_{i} \neq 0}\left(\nu_{i}-q_{i}\right)\right)=\exp \left(2 \pi i s_{g}\right)
$$

Assumption: We will assume that in the data $(f, G, \rho),\langle J\rangle \subset \rho(G)$, when considering duals on the level of $D(k[G])$-modules. Going to the algebra level we postulate that the $G$-Frobenius algebra structures above are Euler or quasi-Euler with fixed Eulerization.

Remark 3.9. The condition above holds for $G_{\max }$, so if it does not hold for a subgroup $H \subset G_{\max }$, then if we are in a quasi-Euler case with fixed Eulerization, we can enlarge $H$ to $G_{\max }$ and perform the dualization for the $G_{\max }$-Frobenius algebra and then reduce to the $H$-Frobenius sub-algebra or the respective $D(k[H])$ module.

3.4.2. The dual $k$-module. Given the $G$-Frobenius algebra $G M_{f}$ its dual $k-$ module is defined as

$$
\check{A}_{g}=A_{g j^{-1}}=M_{\left.f\right|_{\mathrm{Fix}\left(g j^{-1}\right)}}
$$

where $j$ is the group element defining the exponential of the grading operator $Q$ via $\rho(j)=\exp (2 \pi i Q)$.

3.4.3. The dual $D(k[G])$-module. The $G$-module structure is given by pulling back taction and scaling by $\chi$. In the case of a singularity the character $\chi$ is determined by a choice of sign function $\sigma \in \operatorname{Hom}(G, \mathbb{Z} / 2 \mathbb{Z})$ given by $\chi(g)=$ $(-1)^{\sigma(g)} \operatorname{det}(g)$. If we denote the $G$-action on $\check{A}$ by $\check{\varphi}$ then using the $k$-module isomorphism $M: A_{g} \rightarrow A_{g j^{-1}}$

$$
\check{\varphi}(g)\left(\check{a}_{h}\right):=\chi(g) M \varphi(g) M^{-1}\left(\check{a}_{h}\right) \in \check{A}_{h g h^{-1}}, \quad \text { for } \check{a}_{h} \in \check{A}_{g}
$$

or if we denote $M(a)=: \check{a}$ and fix $\sigma \in \operatorname{Hom}(G, \mathbb{Z} / 2 \mathbb{Z})$ then for $\check{a} \in \check{A}_{h}$

$$
\check{\varphi}(g)(\check{a}):=(-1)^{\sigma(g)} \operatorname{det}(g)(\varphi(\check{g})(a)) \in \check{A}_{g h g^{-1}}
$$

Using equation (3.8) for $\check{a}_{h}=M\left(a 1_{h j^{-1}}\right) \subset \check{A}_{h}$

$$
\check{\varphi}(g)\left(\check{a}_{h}\right)=\check{\varphi}(g) M\left(a 1_{h j^{-1}}\right)
$$

$$
=\left.\epsilon\left(g, h j^{-1}\right)(-1)^{\sigma(g)(\sigma(h)+\sigma(j)+1)} \operatorname{det}(g)\right|_{\mathrm{Fix}\left(h j^{-1}\right)} M\left(a 1_{g h g^{-1} j^{-1}}\right)
$$

Remark 3.10. If $\operatorname{det}(g)=(-1)^{\sigma(g)}$ then $\chi$ is trivial. This means that the dual and the $G$-Frobenius algebra have the same invariants.

Lemma 3.4. $\check{1}_{e}$ is invariant if and only if $G M_{f}$ is G-Euler.

Proof. Since $\operatorname{Fix}(j)=\emptyset$ as $f$ can be chosen to contain no linear terms (the linear terms would actually only add Eigenspaces of Eigenvalue one) the condition

$$
\check{\varphi}(g)\left(\check{1}_{e}\right)=\check{1}_{e}
$$


reads

$$
\forall g \in G: \epsilon\left(g, j^{-1}\right)(-1)^{\sigma(g)(\sigma(j)+1)} .
$$

This is precisely the condition to be $G$-Euler of Lemma 3.3.

Corollary 3.2. Unless $\forall g \in G:\left.\epsilon\left(g, j^{-1}\right)(-1)^{\sigma(g)(\sigma(j)+1)} \operatorname{det}(g)\right|_{\text {Fix }(j)}=1$ there is no Frobenius structure on the $G$ invariants of $\breve{G} M_{f}$ for $G M_{f}$ with these invariants.

Proof. Without this condition there will be no invariant unit for the $(a, c)$ ring since for non-trivial grading $\check{1}_{e}$ is the only element of bi-degree $(0,0)$.

Assumption: Due to the content of the lemma, we will only consider taking the invariants of a the dual of a fixed $D(k[G])$ module structure on $G M_{f}$ if it is $G$-Euler.

3.4.4. The bi-grading for the dual. The bi-grading for the dual is given by the general formula $(2.3)$

$$
\check{\mathcal{Q}}(\check{a})=Q(a)+\check{s}_{g} \quad \overline{\check{\mathcal{Q}}}:=Q\left(a_{g}\right)+\overline{\check{s}}_{g} \quad \text { for } \check{a}_{g} \in \check{A}_{g}
$$

In the notation of 3.2.3 this reads as

$\check{s}_{g}:=s_{g j^{-1}}-d=\sum_{i: \nu_{i}\left(g j^{-1}\right) \neq 0}\left(\nu_{i}(g)+\left(1-q_{i}\right)+\Theta\left(q_{i}-\nu_{i}\right)-q_{i}\right)-\sum_{i} 1-2 q_{i}$ and $\check{\bar{s}}_{g}:=\bar{s}_{g j^{-1}}=\sum_{i: \nu_{i}\left(g j^{-1}\right) \neq 0}\left(1-\left(\nu_{i}(g)+\left(1-q_{i}\right)+\Theta\left(q_{i}-\nu_{i}\right)\right)-q_{i}\right)$ and thus

$$
\begin{aligned}
\check{s}_{g} & =\sum_{\nu_{i}\left(g j^{-1}\right) \neq 0}\left(\nu_{i}(g)+\Theta\left(q_{i}-\nu_{i}\right)\right)-d_{g j^{-1}} \\
\check{s}_{g} & =\sum_{\nu_{i}\left(g j^{-1}\right) \neq 0} \Theta\left(q_{i}-\nu_{i}\right)-\nu_{i}(g)
\end{aligned}
$$

Remark 3.11. An element in $\check{A}_{g}$ has anti-diagonal grading $(-q, q)$ if and only if $Q\left(a_{g}\right)=\frac{1}{2} d_{g j^{-1}}$

3.4.5. The metric. The metric is as in the general case the pulled back metric. It will have group degree $j^{2}$ and will be homogenous of bi-degree $(-d, d)$.

3.4.6. The degenerate $G$-Frobenius structure. As remarked previously, for the dual $D(k[G])$ module one cannot expect a $G$-Frobenius structure, but what we called a degenerate $G$-Frobenius algebra of group degree $j$, which induces a $C(G)$ graded Frobenius structure on the invariants, in the sense of [JKK].

3.5. Mirror symmetry for singularities. In the framework of mirror symmetry a quasi-homogenous function $f$ with an isolated singularity is considered as a Landau-Ginzburg $B$-Model and hence has as a $(c, c)$ ring the $(c, c)$ realization of $M_{f}$ and has a trivial $(a, c) \operatorname{ring} A_{1}$.

Definition 3.8. We call a $G$ Euler orbifold $G$-Frobenius algebra A, together with a degenerate $G$-Frobenius algebra of degree $j$ on $\check{A}$ a model for the mirror dual of a singularity $M_{f}$ if the $G$ invariants of $A$ are spanned by $1 \in A$ and the $G$-invariants of $\check{A}$ are the $(a, c)$ realization.

We also just say in short $(\check{A})^{G}$ is the mirror dual to $M_{f}$.

We call two $G$ Euler orbifold $G$-Frobenius algebra $A$ and an $H$ Euler orbifold $H$-Frobenius $B$, together with a degenerate $G$-Frobenius algebra of degree $j$ on $\check{A}$ and a degenerate $H$-Frobenius algebra of degree $j^{\prime}$ on $\check{B}$ mirror dual pair if the $A^{G}=(\check{B})^{H}$ and $(\check{A})^{G}=B^{H}$. In short we say, $A$ and $B$ are mirror dual. 


\begin{tabular}{|c|c|c|c|c|c|}
\hline$M_{f}$ & restriction & $G$ & $\sigma$ & $G M_{f}^{G}$ & $\left(\check{G} M_{f}\right)^{G}$ \\
\hline$A_{n}$ & & $\mathbb{Z} /(n+1) \mathbb{Z}$ & 0 & $\overrightarrow{A_{1}}$ & $A_{n}$ \\
\hline$A_{2 n-1}$ & & $\mathbb{Z} /(n+1) \mathbb{Z}$ & 1 & $A_{1}$ & $B_{n}$ \\
\hline$A_{2 n-1}$ & & $\mathbb{Z} / 2 \mathbb{Z}$ & 0 & $B_{n}$ & $I_{2}(4)$ \\
\hline$A_{2 n-1}$ & $n$ odd for dual & $\mathbb{Z} / 2 \mathbb{Z}$ & 1 & $D_{n+1}$ & $A_{1}$ \\
\hline$A_{2 n-1}$ & & $\mathbb{Z} / n \mathbb{Z}$ & 0 & $I_{2}(4)$ & $B_{n}$ \\
\hline$D_{n+1}$ & & $\mathbb{Z} /(2 n \mathbb{Z})$ & 0 & $A_{1}$ & $A_{2 n-1}$ \\
\hline$D_{n+1}$ & $n$ even & $\mathbb{Z} / n \mathbb{Z}$ & 0 & $I_{2}(4)$ & $B_{n}$ \\
\hline$D_{n+1}$ & $n$ odd & $\mathbb{Z} / n \mathbb{Z}$ & 0 & $A_{1}$ & $D_{n+1}$ \\
\hline$D_{n+1}$ & & $\mathbb{Z} / 2 \mathbb{Z}$ & 0 & $B_{n}$ & $I_{2}(4)$ \\
\hline$D_{n+1}$ & $n$ odd for dual & $\mathbb{Z} / 2 \mathbb{Z}$ & 1 & $A_{2 n-1}$ & $I_{2}(4)$ \\
\hline$A_{k_{1}-1} \otimes \cdots \otimes A_{k_{n}-1}$ & $k_{i}$ coprime & $\mathbb{Z} / k_{1} \mathbb{Z} \times \ldots \mathbb{Z} / k_{n} \mathbb{Z}$ & 0 & $A_{1}$ & $A_{k_{1}-1} \otimes \cdots \otimes A_{k_{n}-1}$ \\
\hline$E_{6}$ & & $\mathbb{Z} / 3 \mathbb{Z} \times \mathbb{Z} / 4 \mathbb{Z}$ & 0 & $A_{1}$ & $E_{6}$ \\
\hline$E_{7}$ & & $\mathbb{Z} / 9 \mathbb{Z}$ & 0 & $A_{1}$ & $E_{7}$ \\
\hline$E_{8}$ & & $\mathbb{Z} / 3 \mathbb{Z} \times \mathbb{Z} / 5 \mathbb{Z}$ & 0 & $A_{1}$ & $E_{8}$ \\
\hline
\end{tabular}

TABLE 1. Since all groups are cyclic $\epsilon \equiv 0, \operatorname{Hom}(G, \mathbb{Z} / 2 \mathbb{Z})=e$ or

$\operatorname{Hom}(G, \mathbb{Z} / 2 \mathbb{Z})=\mathbb{Z} / 2 \mathbb{Z}$ defining the entry in the column $\sigma$. The conditions for the duals are the condition to be be quasi Euler.

Constructions for mirror pairs come form the orbifold mirror philosophy.

\section{A MIRROR THEOREM FOR SIMPLE SINGULARITIES AND OTHER EXAMPLES}

In this section, we calculate the orbifolds and duals in several examples. We will consider the first example in the greatest detail and then leave slightly more details to the reader as we continue with to make the text more concise.

The main result of this is the following Theorem whose proof follows from the calculations below which are collected in the table 4 .

Theorem 4.1. Let $f$ be one of the simple singularities $A_{n}, D_{n}, E_{6}, E_{7}$ and $E_{8}$ or a Pham singularity with coprime powers, let $J$ be the exponential grading operator and $\Gamma:=\langle j\rangle$ with $\rho(j)=J$. Then there is a projectively unique maximally nondegenerate degenerate $\Gamma$-Frobenius algebra structure of degree $j$ on $\Gamma \check{M}_{f}$. Moreover the invariants of the $G$-Frobenius algebra $G M_{f}$ are one dimensional and yield the Frobenius algebra $A_{1}$, while the invariants of the $\Gamma \check{M}_{f}$ are isomorphic as a bi-graded Frobenius algebra to $M_{f}^{(a, c)}$.

In short: the $A, D, E$ singularities are mirror self dual in the sense $\left(\Gamma \check{M}_{g}\right)^{\Gamma}$ is the mirror dual.

It would be tempting to conjecture that if $\Gamma$ is the group generated be the grading operator then $\Gamma \breve{M}_{f}^{\Gamma}$ is the mirror dual. This is however not true as the example of the elliptic singularity $P_{8}$ or a Pham singularity of non-coprime exponents such as $x_{0}^{5}+\cdots+x_{4}^{5}$ below show. One obstruction is that $\Gamma M_{f}^{\Gamma}$ is more than onedimensional. If $\Gamma M_{f}^{\Gamma} \simeq A_{1}$ we would however expect that $\Gamma \check{M}_{f}{ }^{\Gamma}$ is the dual. 


\begin{tabular}{c|c|c|c|c|c}
$T$ & $G$ & $H$ & $K=G / H$ & $\left((T / H)^{H},(T / H)^{H}\right)$ & $\left.((T / H) /(K))^{K},((T / \check{H}) / K)^{K}\right)$ \\
\hline$A_{2 n-1}$ & $\mathbb{Z} /(2 n \mathbb{Z})$ & $\mathbb{Z} / 2 \mathbb{Z}$ & $\mathbb{Z} / n \mathbb{Z}$ & $\left(D_{n+1}, A_{1}\right)$ & $\left(A_{1}, D_{n+1}\right)$ \\
$n$ odd & $\mathbb{Z} /(2 n \mathbb{Z})$ & $\mathbb{Z} / 2 \mathbb{Z}$ & $\mathbb{Z} / n \mathbb{Z}$ & $\left(B_{n}, I_{2}(4)\right)$ & $\left(I_{2}(4), B_{n}\right)$ \\
$A_{2 n-1}$ & $\mathbb{Z}$ & $\left(B_{n}, I_{2}(4)\right)$ & $\left(I_{2}(4), B_{n}\right)$ \\
$D_{n+1}$ & $\mathbb{Z} /(2 n \mathbb{Z})$ & $\mathbb{Z} / 2 \mathbb{Z}$ & $\mathbb{Z} / n \mathbb{Z}$ & $\left(F_{4}, I_{2}(4)\right)$ & $\left(I_{2}(4), F_{4}\right)$ \\
$n$ even & $\mathbb{Z} / 3 \mathbb{Z} \times \mathbb{Z} / 4 \mathbb{Z}$ & $e \times \mathbb{Z} / 2 \mathbb{Z}$ & $\mathbb{Z} / 3 \mathbb{Z} \times \mathbb{Z} / 2 \mathbb{Z}$ &
\end{tabular}

TABLE 2. Mirror pairs from orbifold mirror philosophy ${ }^{3}$

Conjecture 4.1. For an insolated singularity $f$, let $\Gamma$ be the group generated by the exponential grading operator $J$ if $\Gamma M_{f}^{\Gamma} \simeq A_{1}$ then $\Gamma \check{M}_{f}^{\Gamma}$ is the mirror dual.

Also from the explicit calculations below, we obtain that.

Theorem 4.2. The orbifold mirror philosophy holds and produces mirror pairs for the cases self-dual cases with the group $G=G_{\max }=\Gamma$ the group generated by the exponential grading operator and $H=e$ and also for the listed in table 4

Remark 4.1. It is interesting to note that for $B_{n}$ in either of its usual descriptions of folding $A_{2 n-1}$ or $D_{n+1}$ we obtain a non-trivial (a,c) ring which is $I_{2}(4)$. The same holds true for $F_{4}$ This feature seems to distinguish $B_{n}, F_{4}$ as simple boundary singularities.

Remark 4.2. For a given Coxeter group $W$ from the list $A_{n}, B_{n}, D_{n}, E_{6}, E_{7}, E_{8}$, $H_{3}, H_{4}, F_{4}, G_{2}$ and $I_{2}(k)$ We denote by $W$ denotes the Frobenius algebra for the corresponding Coxeter group. For the definition of the respective Frobenius manifolds we refer to $[\mathrm{Du}]$.

Notice that we also use $B_{2}=I_{2}(4)$.

4.1. The case of $A_{n}$. The $A_{n}$ singularity is given by the function $f:=x^{n+1}$. The maximal symmetry group is given by $G:=G_{\max }=\mathbb{Z} /(n+1) \mathbb{Z}$. Set $\zeta_{n}:=$ $\exp \left(2 \pi i \frac{1}{n+1}\right)$ then the exponential grading operator is $J=\zeta_{n} i d$ and $G=\langle j\rangle$ with $\rho(j)=J$.

\subsubsection{The $G$-graded $k$-module $G M_{f}$. Since}

$$
\text { Fix }_{j^{i}}= \begin{cases}\mathbb{C} & \text { if } i=0 \\ 0 & \text { else }\end{cases}
$$

as $k$-modules $M_{f}=A_{n}$ and $M_{\left.f\right|_{0}}=k=A_{1}$, where $A_{k}$ denotes the Frobenius algebra of the $A_{k}$ singularity.

The $k$-module $G M_{f}$ is given by

$$
G M_{f}:=\bigoplus_{i=0}^{n+1} A_{j^{i}}, \quad A_{j^{i}}:=\left\{\begin{array}{ll}
A_{n} & i=0 \\
A_{1} & i=1, \ldots, n+1
\end{array}, \quad G M_{f}=A_{n} \oplus A_{1} \oplus \cdots \oplus A_{1}\right.
$$

\footnotetext{
${ }^{3}$ For the first pair we choose $\sigma=1$ for $\mathbb{Z} /(n+1) \mathbb{Z}$ which restricts to $\sigma=1$ for $\mathbb{Z} / 2 \mathbb{Z}$ and $\sigma=0$ for $\mathbb{Z} / n \mathbb{Z}$ For the second and pair we used the embedding of $B_{n}$ into $A_{2 n-1}$ as a subalgebra in the untwisted sector and for the last pair the embedding of $B_{n}$ into $D_{n+1}$ as a boundary singularity to calculate the last column. For this we consider the respective cyclic submodules over the sub-algebra corresponding to $B_{n}$ in the untwisted sector.
} 
We denote the generator of the $j^{i}$ twisted sector $A_{j^{i}}$ by $1_{j^{i}}$ and have the representation

$$
\rho\left(j^{k}\right)=\zeta_{n+1}^{i}=\exp \left(2 \pi i \frac{k}{n+1}\right)=\exp \left(2 \pi i \nu\left(j^{k}\right)\right) \quad \nu\left(j^{k}\right)=\frac{k}{n+1}
$$

Remark 4.3. We wish to point out that the notation $A_{g}$ for $g \in G$ is the standard notation for the twisted sectors of a $G$-Frobenius algebra $A$, while the notation $A_{n}$ with $n \in \mathbb{N}$ is the standard notation for the Frobenius algebra of the $A_{n}$ singularity. We hope that this will not lead to confusion, since one index is a natural number and the other index an element in a finite group.

4.1.2. The grading. The grading is determined by the shifts

$$
s_{j^{i}}^{+}=\left\{\begin{array}{ll}
0 & \text { if } i=0 \\
\frac{n-1}{n+1} & \text { if } i \in\{1, \ldots, n\}
\end{array}, \quad s_{j^{i}}^{-}= \begin{cases}0 & \text { if } i=0 \\
\frac{2 i-n-1}{n+1} & \text { if } i \in\{1, \ldots, n\}\end{cases}\right.
$$

Thus:

$$
s_{j^{i}}= \begin{cases}0 & \text { if } i=0 \\ \frac{i-1}{n+1} & \text { if } i \neq 0\end{cases}
$$

4.1.3. The super-grading. The choices of super-gradings ${ }^{\sim}$ are determined by a choice of $\sigma \in \operatorname{Hom}(\mathbb{Z} /(n+1) \mathbb{Z}, \mathbb{Z} / 2 \mathbb{Z})$ by

$$
\tilde{1}_{g} \equiv\left|N_{g}\right|+\sigma(g)
$$

where

$$
\left|N_{j^{i}}\right|= \begin{cases}0 & \text { if } i=0 \\ 1 & \text { if } 1 \leq i \leq n\end{cases}
$$

Now if $n=2 m$ is even, $\operatorname{Hom}(\mathbb{Z} /(2 m+1) \mathbb{Z}, \mathbb{Z} / 2 \mathbb{Z})=e$, so $\sigma\left(j^{i}\right) \equiv 0$.

If $n=2 m-1$ is odd, $\operatorname{Hom}(\mathbb{Z} /(2 m) \mathbb{Z}, \mathbb{Z} / 2 \mathbb{Z})=\mathbb{Z} / 2 \mathbb{Z}$ and there are two choices for $\sigma$, either $\sigma\left(j^{i}\right) \equiv 0$ or $\sigma\left(j^{i}\right) F \equiv i \bmod 2$.

Let us fix $\sigma \in \operatorname{Hom}(\mathbb{Z} /(n+1) \mathbb{Z}, \mathbb{Z} / 2 \mathbb{Z})$.

\subsubsection{The bi-grading.}

$$
(\mathcal{Q}, \overline{\mathcal{Q}})\left(1_{j^{i}}\right)= \begin{cases}(0,0) & \text { for } i=0 \\ \left(\frac{i-1}{n+1}, \frac{n-i}{n+1}\right) & \text { else }\end{cases}
$$

Remark 4.4. The elements with a $(q, q)$ grading are the elements $1, z, \ldots, z^{n-1} \in$ $A_{e}$ and $1_{\frac{n+1}{2}}$ in the case that $n=2 m-1$ is odd. The latter element is not invariant under the whole group $\mathbb{Z} /(2 m) \mathbb{Z}$, but is is invariant under the subgroup $\mathbb{Z} / 2 \mathbb{Z}$ as we will show in $\S 4.5$ below.

4.1.5. The $G$-action. We have already fixed $\sigma \in \operatorname{Hom}(\mathbb{Z} /(n+1) \mathbb{Z}, \mathbb{Z} / 2 \mathbb{Z})$.

Since $\mathbb{Z} /(n+1) \mathbb{Z}$ is abelian, its action is determined by a choice of discrete torsion $\epsilon$ by the trace axiom

$$
\epsilon(g, h):=\varphi_{g, h}(-1)^{\sigma(g) \sigma(h)} \operatorname{det}(g) \operatorname{det}\left(\left.g^{-1}\right|_{\mathrm{Fix}(h)}\right)
$$

now since $\epsilon\left(j^{i}, j^{k}\right)=\epsilon(j, j)^{i+k}=1^{i+k}=1$, we find that $\epsilon \equiv 1$ and this implies that

$$
\varphi_{j^{i}, j^{k}}= \begin{cases}1 & \text { if } k=0 \\ (-1)^{\sigma\left(j^{i}\right) \sigma\left(j^{k}\right)} \zeta^{-i} & \text { if } k \in\{1, \ldots, n\}\end{cases}
$$


4.1.6. The metric. After the necessary re-scaling, the metric is given by

$$
\eta\left(z^{i}, z^{k}\right)=\delta_{i+k, n-1} \quad \eta\left(1_{j^{i}}, 1_{j^{k}}\right)=\delta_{i+k, n+1}\left((-1)^{\tilde{j} i} \zeta\right)^{i / 2} z^{n-1}
$$

4.1.7. The $G$-Frobenius structure. Using the reconstruction Theorem we have to find a cocycle $\gamma$ compatible with the action defined by $\varphi$ above and the grading.

From the general considerations we know $\gamma_{j^{i}, j^{n-1-i}} \in A_{e}$ and $\operatorname{deg}\left(\gamma_{j^{i}, j^{n-1-i}}\right)=$ $d-d_{j^{i}}=\frac{n-1}{n+1}$ which yields

$$
\gamma_{j^{i}, j^{n-1-i}}=\left((-1)^{\tilde{j} i} \zeta^{i}\right)^{1 / 2} \rho=\left((-1)^{\tilde{j}} \zeta\right)^{i / 2} z^{n-1}
$$

for the other $\gamma$ notice that $\operatorname{deg}\left(1_{j^{i}}\right)+\operatorname{deg}\left(1_{j^{k}}\right)=\frac{i+k-2}{n+1}$ while $\operatorname{deg}\left(1_{j^{i+j}}\right)=\frac{i+k-1}{n+1}$ if $i+k \neq n+1$, but there is no element of degree $\frac{1}{n+1}$ in $A_{j^{i+k}}$ for $i+k \neq n+1$, so that the respective multiplication must yield zero if the condition is not met.

Hence

$$
\gamma_{j^{i}, j^{k}}= \begin{cases}\left((-1)^{\tilde{j}} \zeta\right)^{i / 2} z^{n-1} & \text { for } i+k=n+1 \\ 0 & \text { else }\end{cases}
$$

4.1.8. The $G$-invariants. Regardless of the choice of $\sigma$ the only invariant of $\mathbb{Z} /(n+1) \mathbb{Z} M_{z^{n+1}}$ is the identity $1 \in A_{e}$.

Proposition 4.1. The $\mathbb{Z} /(n+1) \mathbb{Z}$ invariants of the $D(k[G])$-module $\mathbb{Z} /(n+1) \mathbb{Z} M_{z^{n+1}}$ are spanned by $1 \in A e$ and thus any $\mathbb{Z} /(n+1) \mathbb{Z}$-Frobenius algebras built on $\mathbb{Z} /(n+1) \mathbb{Z} M_{z^{n+1}}$ has as its invariants the Frobenius algebra $A_{1}$.

Remark 4.5. This is the expected result since the dual of $\left(A_{n}, A_{1}\right)$ is $\left(A_{1}, A_{n}\right)$ according to $[\mathrm{V}, \mathrm{IV}]$.

4.1.9. The dual $G$-graded $k$-module. The dual $G$-graded $k$-module $\breve{G} M_{f}:=$ $\bigoplus_{g \in \mathbb{Z} /(n+1) \mathbb{Z}} \check{A}_{g}$ is given by

$$
\check{A}_{j^{-i}}:=\left\{\begin{array}{ll}
A_{1} & i \in\{0, \ldots, n-1\} \\
A_{n} & i=n
\end{array} \check{G Q_{f}=A_{1} \oplus \cdots \oplus A_{1} \oplus A_{n}}\right.
$$

here again the remark 4.3 applies.

Remark 4.6. Notice that it is convenient to choose the generator $j^{-1}$ for the group $\mathbb{Z} /(n+1) \mathbb{Z}$ instead of $j$.

4.1.10. The dual $D(k[G])$ module. The $G$-action on the $\check{A}_{j^{-k}}$ is given by:

$\check{\varphi}_{j^{-i}, j^{-k}}=\varphi_{j^{-i}, j^{-(k+1)}} \chi\left(j^{-i}\right)= \begin{cases}(-1)^{\sigma\left(j^{-i}\right)} \zeta^{-i} & \text { for } k=n \equiv-1 \quad \bmod (n+1) \\ (-1)^{\sigma\left(j^{-i}\right)\left(\sigma\left(j^{-(k+1)}\right)+1\right)} & \text { else }\end{cases}$

4.1.11. The bi-grading. The bi-grading is given by

$$
(\check{\mathcal{Q}}, \overline{\mathcal{Q}})\left(\check{1}_{j-k}\right)= \begin{cases}\left(-\frac{k}{n+1}, \frac{k}{n+1}\right) & k \in\{0,1, \ldots, n-1\} \\ \left(-\frac{n-1}{n+1}, 0\right) & \text { for } k=n\end{cases}
$$

Proposition 4.2. In the case that $\sigma \equiv 0$ the $\mathbb{Z} /(n+1) \mathbb{Z}$-invariants of $\mathbb{Z} /(n+1) \mathbb{Z} M_{z^{n+1}}=$ $\left(\mathbb{Z} /(n+1) \mathbb{Z} A_{n}\right)^{\vee}$ are the linear subspace

$$
\left\langle\check{1}_{e}, \ldots, \check{1}_{j^{-(n-1)}}\right\rangle
$$

This subspace is isomorphic as a graded $k$-module to $A_{n}$. 
In the case that $n=2 m-1$ is odd and $\sigma\left(j^{i}\right) \equiv i \bmod 2$ the $\mathbb{Z} /(n+1) \mathbb{Z}$ invariant subspace is

$$
\left\langle\check{1}_{e}, \check{1}_{j^{-2}}, \ldots, \check{1}_{j^{-2 m}}\right\rangle
$$

This subspace is isomorphic as a graded $k$-module to the $(a, c)$ realization of the sub-k-module $B_{m} \subset A_{2 m-1}$

4.1.12. The metric on the dual $D(k[G])$ algebra. The metric is, after rescaling the generators by a non-zero factor given by the formulas

$$
\begin{aligned}
\check{\eta}\left(1_{j^{-i}}, 1_{j^{-k}}\right) & =\delta_{i+j, n-1} \text { for } i, j \in\{0, \ldots, n-1\} \\
\check{\eta}\left(z^{i} 1_{j^{-n}}, z^{k} 1_{j^{-n}}\right) & =\delta_{i+j, n-1} \\
\check{\eta}\left(1_{j^{-i}}, z^{k} 1_{j^{-n}}\right) & =\check{\eta}\left(z^{k} 1_{j^{-n}}, 1_{j^{-i}}\right)=0 \text { for } i \in\{0, \ldots, n-1\}
\end{aligned}
$$

4.1.13. The degenerate $G$-Frobenius algebra structure. There is a multiplication compatible with the bi-grading. It is unique up to scaling of the generators and is given by

$$
\begin{aligned}
\check{1}_{j^{-i}} \check{1}_{j^{-k}} & = \begin{cases}1_{j^{-(i+k)}} & \text { if } i+k \leq n-1 \\
0 & \text { if } i+k \geq n\end{cases} \\
\check{1}_{j^{-i}} \check{1}_{j^{-n}} & =0 \text { for } i \in\{0, \ldots, n-1\}
\end{aligned}
$$

The following statement is straightforward.

Lemma 4.1. This multiplication renders the metric, invariant i.e. it satisfies $\check{\eta}(\check{a}, \breve{b} \check{c})=\check{\eta}(\check{a}, \breve{b} \check{c})$. Furthermore $\check{\eta}$ is the projectively unique non-degenerate pairing compatible with the bi-grading and the above multiplication is the projectively unique maximally non-degenerate multiplication rendering the metric invariant.

Remark 4.7. The multiplication above is not compatible with the grading and group grading. But changing the equation (4.2) to

$$
z^{n-1-i} \check{1}_{j^{-n}} z^{n-1-j} \check{1}_{j^{-n}}=0
$$

yields a multiplication that is (a) compatible with the bi-grading (b) compatible with the group grading and (c) compatible with the $G$-module structure and thus is compatible with the $D(k[G])$-module. This multiplication does not, however, render the pairing $\check{\eta}$ invariant.

Lemma 4.2. The multiplication of Remark 4.7 is the projectively unique maximally non-degenerate multiplication which is compatible with the $D(k[G])$-module structure, i.e. turn the $D(k[G])$ module into a $D(k[G])$ module and co-module algebra.

Remark 4.8. The metric $\check{\eta}^{\prime}$ given by

$$
\check{\eta}^{\prime}(\check{a}, \check{b}):= \begin{cases}\check{\eta}(\check{a}, \check{b}) & \text { for } \check{a} \in A_{j^{-i}}, \check{b} \in A_{j^{-k}} \quad i, k \in\{0, \ldots, n-1\} \\ 0 & \text { else }\end{cases}
$$

is invariant w.r.t. the multiplication of Remark 4.7. 
Remark 4.9. The multiplication of Remark 4.7 together with the metric of Remark 4.8 which contains degenerate elements and has a non-trivial annihilator of the whole algebra is reminiscent of the appearance of so-called Ramond sectors in the theory of spin-curves [JKV, PV, P]. For a discussion, see $§ 5.1$ below.

Collecting the results from above yields:

Proposition 4.3. In the case $\sigma \equiv 0$, the $\mathbb{Z} /(n+1) \mathbb{Z}$ invariants of $\mathbb{Z} /(n+1) \mathbb{Z} M_{z^{n+1}}=$ $\mathbb{Z} /(n+1) \mathbb{Z} A_{n}$ together with the multiplication of Remark 4.7 and the metric of Remark 4.8 are a degenerate $G$-Frobenius algebra of charge $j$ which is a projectively unique maximally non-degenerate algebra. The Frobenius algebra given by the invariants with the grading $\overline{\mathcal{Q}}$ is isomorphic to $A_{n}$ as graded Frobenius algebras.

As bi-graded Frobenius algebras, the invariants of the dual are the $(a, c)$ realization or $A_{n}:\left(\mathbb{Z} /(n+1) \mathbb{Z} A_{n}^{(c, c)}\right)^{\mathbb{Z} /(n+1) \mathbb{Z}}=A_{n}^{(a, c)}$.

In this sense, $\left(\mathbb{Z} /(n+1) \mathbb{Z} A_{n}\right)^{\mathbb{Z} /(n+1) \mathbb{Z}}=A_{n}$ is the mirror and $A_{n}$ is mirror self-dual.

In the case $n=2 m-1$ and $\sigma\left(j^{i}\right) \equiv i \bmod 2 \mathbb{Z} /(n+1) \mathbb{Z} A_{n}$ together with the multiplication of Remark 4.7 and the metric of Remark 4.8 are a degenerate $G$ Frobenius algebra of degree $j$ which is the projectively unique maximally nondegenerate algebra. This Frobenius algebra of the group invariants is isomorphic to the Frobenius sub-algebra $B_{m} \subset A_{n}$ as graded Frobenius algebras. In terms of the bi-grading the invariants dual of $\mathbb{Z} /(n+1) \mathbb{Z} A_{n}^{(c, c)}$ with non-trivial $\sigma$ is $B_{m}^{a, c}$.

4.2. The case of $A_{2 n-1}$ with a $\mathbb{Z} / 2 \mathbb{Z}$ action. In the case of $A_{2 n-1}$, we can restrict ourselves to the action of the subgroup $\mathbb{Z} / 2 \mathbb{Z} \subset \mathbb{Z} /(2 n \mathbb{Z})$, generated by $j^{n}=:-1$ which acts on $z$ by $z \mapsto-z$.

We now consider the singularity $A_{2 n-1}$ with the group of symmetries $\mathbb{Z} / 2 \mathbb{Z}$.

4.2.1. The $G$-Frobenius algebras. The data of the bi-graded $D(k[G])$ module can be read of by restricting the data of 4.1 .

There is a unique twisted sector for the element $j^{n}$ and the algebra

$$
A_{e}=A_{2 n-1}, A_{-1}=A_{1}=k \quad \mathbb{Z} / 2 \mathbb{Z} M_{z^{2 n}}=A_{2 n-1} \oplus k
$$

The $G$ action is again determined by the fact that $\mathbb{Z} / 2 \mathbb{Z}$ is cyclic forcing $\epsilon \equiv 1$ and a choice of $\sigma \in \operatorname{Hom}(\mathbb{Z} / 2 \mathbb{Z}, \mathbb{Z} / 2 \mathbb{Z})$

The bi-grading is given by

$$
\varphi_{-1,-1}=(-1)^{\sigma(-1)+1}
$$

$$
s_{-1}^{+}=\frac{n-1}{n}, \quad s_{-1}^{-}=0, \quad s_{-1}=\bar{s}_{-1}=\frac{n-1}{2 n}
$$

The super-grading is given by $\tilde{1}_{-1} \equiv \sigma(-1) \bmod 2$

The metric is

$$
\eta\left(z^{i}, z^{k}\right)=\delta_{i+k, 2 n-2}, \quad \eta\left(1_{-1}, 1_{-1}\right)=1, \quad \eta\left(z^{i}, 1_{-1}\right)=\eta\left(1_{-1}, z^{i}\right)=0
$$

Taking into account the results of [Ka3, Ka4], there is a unique $\mathbb{Z} / 2 \mathbb{Z}$-Frobenius algebra structure with the multiplication

$$
\begin{gathered}
z^{i} \circ z^{k}=z^{i+k} \quad \text { for } i+k \leq 2 n-2, z^{i} \circ z^{k}=0 \quad \text { for } i+k>2 n-2 \\
z^{i} \circ 1_{-1}=\delta_{i, 0} 1_{j^{m-1}} \quad 1 \circ 1_{-1}=1_{-1}, \quad 1_{-1} \circ 1_{-1}=z^{2 n-2} .
\end{gathered}
$$


This multiplication and the metric are compatible with the bi-grading and yield a $\mathbb{Z} / 2 \mathbb{Z}$ Frobenius algebra in both cases.

Notice that since $\operatorname{det}(g)= \pm 1$ the metric will make the invariants into a Frobenius algebra.

In the case of $\sigma\left(j^{m}\right) \equiv 1 \bmod 2$ we obtain as invariants

$$
\left\langle 1, z^{2}, \ldots z^{2(n-1)}, 1_{j^{n}}\right\rangle
$$

The bi-grading of the invariants is diagonal and given by

$$
\left(\left(\frac{1}{n}, \frac{1}{n}\right), \ldots,\left(\frac{n-1}{n}, \frac{n-1}{n}\right),\left(\frac{n-1}{2 n}, \frac{n-1}{2 n}\right)\right) .
$$

In the case $\sigma\left(j^{m}\right) \equiv 0$ the space of invariants is

$$
\left\langle 1, z^{2}, \ldots z^{2(n-1)}\right\rangle
$$

and the multiplication, the metric and the bi-grading is the restriction of the ones above.

Proposition 4.4. In total we obtain,

(1) The $\mathbb{Z} / 2 \mathbb{Z}$ invariants of $\mathbb{Z} / 2 \mathbb{Z} M_{z^{2 m-2}}$ with the choice $\sigma\left(j^{n}\right) \equiv 1 \bmod 2$ are isomorphic as a bi-graded Frobenius algebra to the $(c, c)$ model of $M_{x^{m}+x y^{2}}=$ $D_{m}$.

(2) The $\mathbb{Z} / 2 \mathbb{Z}$ invariants of $\mathbb{Z} / 2 \mathbb{Z} M_{z^{2 m-1}}$ with the choice $\sigma\left(j^{n}\right) \equiv 0 \bmod 2$ are isomorphic as a bi-graded Frobenius algebra to $B_{m}^{(c, c)}$.

Remark 4.10. The result above in which the invariants of the untwisted sector of $A_{2 n-1}$ yield $B_{n}$ is an instance of what is called folding cf. $[\mathrm{Z}]$ and see $\S 5.3$ below.

4.2.2. The dual $D(k[G])$-module. For the dual, we obtain two sectors

$$
\check{A}_{e}=M\left(A_{j^{-1}}\right) \simeq A_{1}, \quad \check{A}_{j^{n}}=M\left(A_{j^{n-1}}\right) \simeq A_{1}
$$

The dual bi-grading is given by

$$
\check{s}_{e}=\bar{s}_{e}=0, \quad \check{s}_{-1}=-\frac{1}{2} \bar{s}_{-1}=\frac{1}{2}
$$

Remark 4.11. In the case, that $\sigma \equiv 0$ or the case that $n$ is odd and $\sigma(j)=$ $\sigma(-1)=-1$ the action is the restriction of the Euler $G$-Frobenius algebra of $\S 4.1$ and is thus quasi-Euler.

In both these cases the dual action is defined and is given by

$$
\check{\varphi}_{-1,1}=(-1)^{\sigma(-1)(\sigma(j)+1)}=1, \quad \bar{\varphi}_{-1,-1}=(-1)^{\sigma(-1)(\sigma(-1)+\sigma(j)+1)}=(-1)^{\sigma(-1)}
$$

Since is $\left(A_{2 n-1}, \mathbb{Z} / 2 \mathbb{Z}\right)$ is not Euler, but only quasi-Euler, we cannot pull back the metric, but due to the grading there is projectively only one compatible homogenous metric.

Proposition 4.5. Projectively there is a Frobenius algebra structure on the duals compatible with the group grading which is isomorphic as a bi-graded Frobenius algebra to the $(a, c)$ realization of the algebra $I_{2}(4)$. In the case that $\sigma \equiv 0$ the invariants are $I_{2}(4)$ and in the case that $n$ is odd and $\sigma(-1)=-1$ the invariants are $A_{1}$. 
4.3. The case of $A_{2 n-1}$ with symmetry group $\mathbb{Z} / n \mathbb{Z}$. In the case of $A_{2 n-1}$, we can also consider the symmetry group $\mathbb{Z} / n \mathbb{Z} \subset \mathbb{Z} /(2 n \mathbb{Z})$ which is generated by $j^{2}$.

Again the group is cyclic and $\epsilon \equiv 1$. In the case that $n$ is even there is only one possible choice of $\sigma \equiv 0$. In the case that $n$ is odd there are two possible choices $\sigma \equiv 0$ or $\sigma\left(j^{2 k}\right) \equiv k$. The later choice is not quasi-Euler, however.

4.3.1. The $G$-Frobenius algebras. The invariants can be read off from $\S 4.1$. For $\sigma \equiv 0$ or for $\sigma\left(j^{2 k}\right) \equiv k$, there are no invariants in the twisted sector and the invariants in the untwisted sector are

The bi-degrees are $(0,0),\left(\frac{1}{2}, \frac{1}{2}\right)$.

$$
\left\langle 1, z^{n}\right\rangle
$$

Proposition 4.6. Projectively there is only one Frobenius algebra structure on these invariants compatible with the group grading which is isomorphic as a bigraded Frobenius algebra to the $(c, c)$ realization of the algebra for the Coxeter group $I_{2}(4)$. This is also the restriction of the respective multiplication on the unique $\mathbb{Z} /(2 n \mathbb{Z})$ Frobenius algebra $\mathbb{Z} /(2 n \mathbb{Z}) M_{z^{2 n}}$.

4.3.2. The dual. We can only consider the quasi-Euler choice $\sigma \equiv 0$.

The linear spaces for the dual $D(k[G])$ module are all one dimensional $\check{A}_{\Lambda^{2 k}}=$ $k \check{1}_{\Lambda^{2 k}}$ and are all invariant.

The bi-degrees are

$$
(\mathcal{Q}, \overline{\mathcal{Q}})\left(\check{1}_{\Lambda^{2 k}}\right)=\left(-\frac{k}{n}, \frac{k}{n}\right)\left(\check{1}_{\Lambda^{2 k}}\right) \quad k \in\{1, \ldots, n-1\}
$$

Proposition 4.7. The dual $\mathbb{Z} / n \mathbb{Z} A_{2 n-1}$ affords a projectively unique graded $\mathbb{Z} / n \mathbb{Z}-$ Frobenius algebra structure with trivial $\mathbb{Z} / n \mathbb{Z}$ action which is equal to the Frobenius algebra of its invariants and is isomorphic to the $(a, c)$ realization of $B_{n}$

4.4. The case of $A_{p-1} \otimes A_{q-1}$ especially $E_{6}$ and $E_{8}$. We will consider the tensor product $A_{p-1} \otimes A_{q-1}$ for coprime $p, q$.

The corresponding quasi-homogenous singularity is given by $f=x^{p}+y^{q}$. For these singularities $q_{x}=\frac{1}{p}, q_{y}=\frac{1}{q}, d=\frac{2(p q-p-q)}{p q}, \mu=p+q$

Since $p$ and $q$ are coprime $G_{\max }=\mathbb{Z} /(p q \mathbb{Z})=\mathbb{Z} / p Z \times \mathbb{Z} / q \mathbb{Z}$ which is generated by the grading operator $\Lambda=\left(\Lambda_{p}, \Lambda_{q}\right)$ in the tensor representation for the symmetry groups of the $A_{n}$ factors.

$$
\rho(\Lambda)=\left(\begin{array}{cc}
\zeta_{p} & 0 \\
0 & \zeta_{q}
\end{array}\right)
$$

where as usual $\zeta_{p}=\exp \left(2 \pi i \frac{1}{p}\right)$ and $\zeta_{q}=\exp \left(2 \pi i \frac{1}{q}\right)$.

\subsubsection{The $G$-Frobenius algebras.}

$$
f_{\Lambda^{i}}= \begin{cases}z^{p}+z^{q} & \text { if } i=0 \\ z^{p} & \text { if } i=r p, r \in\{1, \ldots, q-1\} \\ z^{q} & \text { if } i=r q, r \in\{1, \ldots, p-1\} \\ 0 & \text { else }\end{cases}
$$

in these cases, we get the twisted sectors linearly isomorphic to $A_{p-1} \otimes A_{q-1}, A_{p-1}, A_{q-1}$ and $A_{1}$. The group is cyclic and hence $\epsilon \equiv 0$. In the case that $p q$ is odd, we only 
have the trivial choice $\sigma \equiv 0$. In the case that it is even we also have the possibility to set $\sigma\left(\Lambda^{i}\right) \equiv i$. We will leave the latter case to the reader.

The action is given by

$$
\varphi_{\Lambda^{k}, \Lambda^{i}}= \begin{cases}1 & \text { if } i=0 \\ \zeta_{q}^{-k} & \text { if } i=r p, r \in\{1, \ldots, q-1\} \\ \zeta_{p}^{-k} & \text { if } i=r q, r \in\{1, \ldots, p-1\} \\ \zeta_{q}^{-k} \zeta_{p}^{-k} & \text { else }\end{cases}
$$

and we see that only $1 \in A_{e}$ is invariant.

The grading is given by

$$
\begin{gathered}
s_{\Lambda^{i}}= \begin{cases}0 & \text { if } i=0 \\
\frac{j-1}{q} & \text { if } i=r p=k q+j, r \in\{1, \ldots, q-1\} \\
\frac{j-1}{p} & \text { if } i=r q=k p+j, r \in\{1, \ldots, p-1\} \\
\frac{j-1}{p}+\frac{l-1}{q} & \text { if } i=r p+j=k q+l\end{cases} \\
\bar{s}_{\Lambda^{i}}= \begin{cases}0 & \text { if } i=0 \\
1-\frac{j+1}{q} & \text { if } i=r p=k q+j, r \in\{1, \ldots, q-1\} \\
1-\frac{j+1}{p} & \text { if } i=r q=k p+j, r \in\{1, \ldots, p-1\} \\
2-\frac{j+1}{p}-\frac{l+1}{q} & \text { if } i=r p+j=k q+l\end{cases}
\end{gathered}
$$

Proposition 4.8. The $\mathbb{Z} / p q Z$ invariants of the unique $D(k[G])$ module $\mathbb{Z} / p q Z A_{p-1} \otimes$ $A_{q-1}$ are one dimensional and are thus isomorphic to the Frobenius algebra $A_{1}$.

4.4.2. The dual. The dual action is given by

$$
\check{\varphi}_{\Lambda^{k}, \Lambda^{i}}= \begin{cases}\zeta_{q}^{-k} \zeta_{p}^{-k} & \text { if } i-1=0 \\ \zeta_{p}^{k} & \text { if } i-1=r p, r \in\{1, \ldots, q-1\} \\ \zeta_{q}^{k} & \text { if } i-1=r q, r \in\{1, \ldots, p-1\} \\ & \text { else }\end{cases}
$$

From this we obtain a $p q-p-q+1=(p-1)(q-1)$ dimensional space of invariants spanned by

$$
\left\langle\check{1}_{\Lambda^{i}}\right\rangle \quad i-1 \not \equiv 0 \quad \bmod p \text { and } i-1 \not \equiv 0 \quad \bmod q
$$

The grading is given by

$$
\begin{aligned}
& \check{s}_{\Lambda^{i}}= \begin{cases}0 & \text { if } i=0 \\
-\frac{2(p q-q-p)}{p q} & \text { if } i=1 \\
\frac{j-1}{q}+\frac{2}{p}-2 & \text { if } i-1=r p=k q+j, r \in\{1, \ldots, q-1\} \\
\frac{j-1}{p} \frac{2}{q}-2 & \text { if } i-1=r q=k p+j, r \in\{1, \ldots, p-1\} \\
\frac{j-1}{p}+\frac{l}{q}-2 & \text { if } i-1=r p+j=k q+l\end{cases} \\
& \bar{s}_{\Lambda^{i}}= \begin{cases}0 & \text { if } i=0 \\
0 & \text { if } i=1 \\
1-\frac{j-1}{q} & \text { if } i-1=r p=k q+j, r \in\{1, \ldots, q-1\} \\
1-\frac{j-1}{p} & \text { if } i-1=r q=k p+j, r \in\{1, \ldots, p-1\} \\
2-\frac{j-1}{p}-\frac{l-1}{q} & \text { if } i-1=r p+j=k q+l\end{cases}
\end{aligned}
$$


where we choose $i \in\{0, \ldots, p q-1\}$.

By comparing degrees we arrive at:

Lemma 4.3. Let $i \equiv j \bmod p, j \in 2, \ldots p$ and $i \equiv k \bmod q, k \in\{2, \ldots, q\}$ then the map

$$
\check{1}_{\Lambda^{i}} \mapsto x^{p-j} y^{q-l}
$$

induces an isomorphism of graded vector spaces between the $\mathbb{Z} /$ pqZ invariants of $\left(\mathbb{Z} / p q \mathbb{Z} M_{x^{p}+y^{q}}\right)^{\vee}=\left(\mathbb{Z} / p q \mathbb{Z} A_{p-1} \otimes A_{q-1}\right)^{\vee}$ graded by $\overline{\mathcal{Q}}$ and the graded Milnor ring $A_{p-1} \otimes A_{q-1}$.

Moreover as bi-graded space $\left.\left(\left(\mathbb{Z} / p q \mathbb{Z} A_{p-1} \otimes A_{q-1}\right)\right)^{\vee}\right)^{\mathbb{Z} / p q \mathbb{Z}}$ is the $(a, c)$ realization or the $A$-model of $A_{p-1} \otimes A_{q-1}$.

By comparing the degrees and group degrees, one obtains:

Proposition 4.9. There is a projectively unique maximally non-degenerate degenerate $G-$ Frobenius structure on $\left(\mathbb{Z} / p q \mathbb{Z} A_{p-1} \otimes A_{q-1}\right)^{\vee}$ whose invariants are the mirror dual to $A_{p-1} \otimes A_{q-1}$

Corollary 4.1. If we restrict ourselves to the case $p=3, q=4$, we obtain the mirror to the $E_{6}$ singularity and for $p=3, q=5$ the mirror for the $E_{8}$ singularity.

In the first case the invariants are

$$
\check{1}_{\Lambda^{i}}, i \in\{0,2,3,6,8,11\} \text { corresponding to } 1, x y^{2}, y, y^{2}, x, x y
$$

with bi-degrees

$$
(0,0),\left(-\frac{5}{6}, \frac{5}{6}\right),\left(-\frac{1}{4}, \frac{1}{4}\right),\left(-\frac{1}{2}, \frac{1}{2}\right),\left(-\frac{1}{3}, \frac{1}{3}\right),\left(-\frac{7}{12}, \frac{7}{12}\right)
$$

In the second case the invariants are

$$
\check{1}_{\Lambda^{i}}, i \in\{0,2,3,5,8,9,12,14\} \text { corresponding to } 1, x y^{3}, y^{2}, x, x y^{2}, y, y^{3}, x y
$$

with bi-degrees

$$
(0,0),\left(-\frac{14}{15}, \frac{14}{15}\right),\left(-\frac{2}{5}, \frac{2}{5}\right),\left(-\frac{1}{3}, \frac{1}{3}\right),\left(-\frac{11}{15}, \frac{11}{15}\right),\left(-\frac{1}{5}, \frac{1}{5}\right),\left(-\frac{3}{5}, \frac{3}{5}\right),\left(-\frac{8}{15}, \frac{8}{15}\right)
$$

4.4.3. The case of $E_{6}$ and the relation to $F_{4}$. Using the above calculations we can obtain a mirror pair for $F_{4}$ from $E_{6}$ via the tensor product. For this we use that $E_{6}=\left(A_{2} \otimes A_{3}\right)$ and $G_{\max }=\mathbb{Z} / 3 Z \times \mathbb{Z} / 4 \mathbb{Z}$.

Proposition 4.10. $\left(F_{4}, I_{2}(4)\right)$ and $\left(I_{2}(4), F_{4}\right)$ are a mirror dual pair obtained from the orbifold mirror philosophy for $E_{6}$ with $G_{\max }=\mathbb{Z} / 3 \mathbb{Z} \times \mathbb{Z} / 4 \mathbb{Z}, H=e \times \mathbb{Z} / 2 \mathbb{Z}$ and $G / H=\mathbb{Z} / 3 \mathbb{Z} \times \mathbb{Z} / 2 \mathbb{Z}$.

Proof. Using the group $\mathbb{Z} / 2 \mathbb{Z}$ acting via $e \times \mathbb{Z} / 2 \mathbb{Z}: E_{6} /(\mathbb{Z} / 2 \mathbb{Z})=\left(A_{2} \otimes A_{3}\right) /(e \times$ $\mathbb{Z} / 2 \mathbb{Z})=\left(A_{2} / e \otimes A_{3} /(\mathbb{Z} / 2 \mathbb{Z})\right)$. Thus by the previous calculations:

$$
\left(\left((\mathbb{Z} / 2 \mathbb{Z}) E_{6}\right)^{\mathbb{Z} / 2 \mathbb{Z}},\left(\left((\mathbb{Z} / 2 \mathbb{Z}) E_{6}\right)^{\vee}\right)^{\mathbb{Z} / 2 \mathbb{Z}}\right)=\left(A_{2} \otimes I_{2}(4), A_{1} \otimes I_{2}(4)\right)=\left(F_{4}, I_{2}(4)\right)
$$

For the dual pair with $G_{\max }=\mathbb{Z} / 3 \mathbb{Z} \times \mathbb{Z} / 4 \mathbb{Z}, H=e \times \mathbb{Z} / 2 \mathbb{Z}$ and $G / H=$ $\mathbb{Z} / 3 \mathbb{Z} \times \mathbb{Z} / 2 \mathbb{Z}:$

$$
\begin{array}{r}
\left(\left(A_{2} \otimes A_{3} /(\mathbb{Z} / 2 \mathbb{Z})\right) /(\mathbb{Z} /(3 \mathbb{Z}) \times \mathbb{Z} / 2 \mathbb{Z})\right)^{H}=A_{2} /(\mathbb{Z} / 3 \mathbb{Z}) \otimes\left(\left(A_{3} /(\mathbb{Z} / 2 \mathbb{Z})\right) /(\mathbb{Z} / 2 \mathbb{Z})\right)^{\mathbb{Z} / 2 \mathbb{Z}} \\
=A_{1} \otimes I_{2}(4) \simeq I_{2}(4)
\end{array}
$$




$$
\begin{aligned}
& \left(\left(\left(A_{2} \otimes A_{3} /(\mathbb{Z} / 2 \mathbb{Z})\right) /(\mathbb{Z} /(3 \mathbb{Z}) \times \mathbb{Z} / 2 \mathbb{Z})\right)^{\vee}\right)^{H} \\
& \left.\quad=\left(\left(A_{2} /(\mathbb{Z} / 3 \mathbb{Z})\right)^{\vee}\right)^{\mathbb{Z} / 3 \mathbb{Z}} \otimes\left(\left(\left(A_{3} /(\mathbb{Z} / 2 \mathbb{Z})\right) /(\mathbb{Z} / 2 \mathbb{Z})\right)^{\vee}\right)^{\mathbb{Z} / 2 \mathbb{Z}}=A_{2} \otimes I_{2}(4)\right) \simeq F_{4}
\end{aligned}
$$

4.4.4. Certain Pham singularities. The same reasoning holds true for the Pham singulaties of coprime powers

$$
f=x_{1}^{k_{1}}+\ldots x_{n}^{k_{n}} \text { with } k_{i} \text { pairwise coprime }=A_{k_{1}-1} \otimes A_{k_{n}-1}
$$

Let $\Gamma$ be the group generated by the grading operator then $\Gamma=\mathbb{Z} / k_{1} \mathbb{Z} \times \ldots \mathbb{Z} / k_{n} \mathbb{Z}$

Proposition 4.11. The $\Gamma$ invariants of the $\Gamma M_{f}$ with the choice of trivial $\sigma$ are $A_{1}$ and there is a degenerate maximally non-degenerate multiplication on the dual $\check{\Gamma} M_{f}$ which is projectively unique and the $\Gamma$ invariants of the dual are the $(a, c)$ realization of $M_{f}$.

In other words $\check{\Gamma} M_{f}$ is mirror for $M_{f}$.

4.5. The case of $D_{n}$. Recall that $D_{n+1}=M_{x^{n}+y^{2} z}$ with $q_{1}=q_{x}=\frac{1}{n}, q_{2}=q_{y}=$ $\frac{n-1}{2 n}, d=\frac{n-1}{n}$. For $n>4$ the maximal symmetry group is $G_{\max }=\mathbb{Z} / 2 n \mathbb{Z}=\langle\Lambda\rangle$. The maximal symmetry group in the case $n=4$ is larger $G_{\max }=\mathbb{Z} / 3 \mathbb{Z} \times \mathbb{S}_{3}$, but also contains the group $\mathbb{Z} / 6 \mathbb{Z}$ generated by $\Lambda$. We will make further comments about the case $D_{4}$ below in $\S 4.8 .3$.

If we fix $\zeta_{2 n}:=\exp \left(2 \pi i \frac{1}{2 n}\right)$, then

$$
\begin{gathered}
\Lambda=\left(\begin{array}{cc}
\zeta_{2 n}^{2} & 0 \\
0 & \zeta_{2 n}^{-1}
\end{array}\right) \quad \Lambda^{i}=\left(\begin{array}{cc}
\zeta_{2 n}^{2 i} & 0 \\
0 & \zeta_{2 n}^{-i}
\end{array}\right) \\
\Lambda^{n+1}=\left(\begin{array}{cc}
\exp \left(2 \pi i \frac{1}{n}\right) & 0 \\
0 & \exp \left(2 \pi i \frac{n-1}{2 n}\right)
\end{array}\right)=\exp (2 \pi i Q)=\rho(j)=J
\end{gathered}
$$

This implies that $G_{\max }=\langle J\rangle$ if and only if $n$ is odd.

Since $e$ fixes both $x$ and $y, \Lambda^{l}$ fixes neither $x$ nor $y$ for $l \neq 0, n$ and $\Lambda^{n}$ fixes $x$ but not $y$, we see that the orbifold data is as follows

\begin{tabular}{l|c|c|c|c|c|c|c|c|c}
$g \in \mathbb{Z} /(2 n \mathbb{Z})$ & $f_{g}$ & $M_{f_{g}}$ & $d_{g}$ & $\nu_{1}(g)$ & $\nu_{2}(g)$ & $\frac{1}{2} s_{g}^{+}$ & $\frac{1}{2} s_{g}^{-}$ & $s_{g}$ & $\bar{s}_{g}$ \\
\hline$g=e=\Lambda^{0}$ & $x^{n}+x y^{2}$ & $D_{n+1}$ & $\frac{n-1}{n}$ & 0 & 0 & 0 & 0 & 0 & 0 \\
$g=\Lambda^{l}, 0<l<n$ & 0 & $A_{1}$ & 0 & $\frac{l}{n}$ & $\frac{2 n-l}{n}$ & $\frac{n-1}{2 n}$ & $\frac{l}{2 n}$ & $\frac{l+n-1}{2 n}$ & $\frac{n-1-l}{2 n}$ \\
$g=\Lambda^{n}$ & $x^{n}$ & $A_{n-1}$ & $\frac{n-2}{n}$ & 0 & $\frac{1}{2}$ & $\frac{1}{n}$ & 0 & $\frac{1}{2 n}$ & $\frac{1}{2 n}$ \\
$g=\Lambda^{l}, n<l<2 n-1$ & 0 & $A_{1}$ & 0 & $\frac{l-n}{n}$ & $\frac{2 n-l}{2 n}$ & $\frac{n-1}{2 n}$ & $\frac{l-2 n}{2 n}$ & $\frac{l-n-1}{2 n}$ & $\frac{3 n-1-l}{2 n}$
\end{tabular}

Comparing the degrees, we arrive at:

Proposition 4.12. The only elements of bi-degree $(q, q)$ of the bi-graded $D(k[G])$ module $\mathbb{Z} /(2 n \mathbb{Z}) D_{n+1}$ are the elements of the untwisted sector.

4.6. The $G$ action. Since $\mathbb{Z} /(2 n \mathbb{Z})$ is cyclic there is only one choice of discrete torsion which fixes the choice of $\varphi$ to be $\varphi_{\Lambda^{k}, \Lambda^{l}}=(-1)^{\sigma(k) \sigma(l)} \operatorname{det}^{-1}\left(\Lambda^{k}\right) \operatorname{det}\left(\left.\Lambda^{k}\right|_{F i x_{\Lambda^{l}}}\right)$ which reads

$$
\varphi_{\Lambda^{k}, \Lambda^{l}}= \begin{cases}1 & l=0 \\ (-1)^{\sigma(k) \sigma(l)} \zeta_{2 n}^{-k} & l \neq 0, n \\ (-1)^{\sigma(k) \sigma(n)} \zeta_{2 n}^{k} & l=n\end{cases}
$$


4.6.1. The metric. The pairing on the twisted sectors is given by

$$
\eta\left(1_{\Lambda^{k}}, 1_{\Lambda^{l}}\right)=\left\{\begin{array}{ll}
\delta_{k+l, 2 n} \exp \left(2 \pi i \frac{k}{4 n}\right) & \text { for } k \leq n \\
\delta_{k+l, 2 n} \exp \left(2 \pi i \frac{2 n-k}{4 n}\right) & \text { for } k \leq n
\end{array}, \quad \eta\left(x^{k} 1_{\Lambda^{n}}, x^{k} 1_{\Lambda^{n}}\right)=\delta_{k+l, n-2}\right.
$$

all other pairings are zero except for the pairing on the untwisted sector, which remains the pairing of $D_{n}$.

Lemma 4.4. The $\mathbb{Z} /(2 n \mathbb{Z})$ invariant subspace is $\langle 1\rangle$, thus

$$
\left(D_{n+1} / \mathbb{Z} /(2 n \mathbb{Z})\right)^{\mathbb{Z} /(2 n \mathbb{Z})}=A_{1}
$$

as a graded Frobenius algebra.

4.6.2. The $G$-Frobenius structure. A straightforward calculation shows that:

Proposition 4.13. There is projectively only one G-Frobenius algebra structure compatible with the bi-grading, which is given by

$$
\gamma_{\Lambda^{k}, \Lambda^{l}}= \begin{cases}\delta_{k+l, 2 n} \exp \left(2 \pi i \frac{k}{4 n}\right) \text { for } k \leq n & \\ \delta_{k+l, 2 n} \exp \left(2 \pi i \frac{2 n-k}{4 n}\right) & \text { for } k \leq n \\ \delta_{l, n} 1 & \text { for } k=n\end{cases}
$$

4.6.3. The dual $D(k[G])$ module. The bi-grading is given by

$$
\begin{aligned}
& \check{s}_{\Lambda^{-k}}= \begin{cases}-\frac{k}{2 n} & \text { if } k \in\{0,1,2, \ldots, n-2, n, \ldots, 2 n-1\} \\
-\frac{n-1}{n} & \text { if } k=n-1 \\
\frac{3-2 n}{2 n} & \text { if } k=2 n-1\end{cases} \\
& \bar{s}_{\Lambda^{-k}}= \begin{cases}\frac{k}{2 n} & \text { if } k \in\{0,1,2, \ldots, n-2, n, \ldots, 2 n-1\} \\
0 & \text { if } k=n-1 \\
\frac{1}{2 n} & \text { if } k=2 n-1\end{cases}
\end{aligned}
$$

Lemma 4.5. In the case that $n$ is even, the only elements of bi-grading $(-q, q)$ of the dual $D(k[G])$ module $\mathbb{Z} /(2 n \check{Z}) D_{n+1}$ are

$$
\left\langle\check{1}_{e}, \check{1}_{\Lambda^{-1}}, \ldots, \check{1}_{\Lambda^{-(n-2)}}, y 1_{\Lambda^{-(n-1)}}, 1_{\Lambda^{-n}}, \ldots, 1_{\Lambda^{-(2 n-2)}}, x^{\frac{n-2}{2}} 1_{\Lambda^{-(2 n-1)}}\right\rangle
$$

and in the case that $n$ is odd, the elements of degree $(-q, q)$ of the dual $D(k[G])$ module $\mathbb{Z} /(2 n \check{\mathbb{Z}}) D_{n+1}$ are

$$
\left\langle\check{1}_{e}, \check{1}_{\Lambda^{-1}}, \ldots, \check{1}_{\Lambda^{-(n-2)}}, x^{\frac{n-1}{2}} 1_{\Lambda^{-(n-1)}}, y 1_{\Lambda^{-(n-1)}}, 1_{\Lambda^{-n}}, \ldots, 1_{\Lambda^{-(2 n-2)}}\right\rangle .
$$

4.6.4. The dual $G$-action. The dual $G$-action is given by

$$
\check{\varphi}_{\Lambda^{-k}, \Lambda^{-l}}= \begin{cases}(-1)^{\sigma\left(\Lambda^{k}\right)\left(\sigma\left(\Lambda^{l}\right)+\sigma\left(\Lambda^{n+1}\right)+1\right)} & \text { for } l \notin\{n-1,2 n-1\} \\ (-1)^{\sigma\left(\Lambda^{k}\right) \zeta_{2 n}^{k}} & \text { for } l=n-1 \\ (-1)^{\sigma\left(\Lambda^{k}\right)\left(\sigma(\Lambda)+\sigma\left(\Lambda^{n+1}\right)+1\right)} \zeta_{n}^{k} & \text { for } l=2 n-1\end{cases}
$$

A longer but straightforward calculation shows

Proposition 4.14. For the different choices of $\sigma$ we obtain: 
(1) In the case $\sigma \equiv 0$, the $\mathbb{Z} /(2 n \mathbb{Z})$ invariants of $\left(\mathbb{Z} /(2 n \mathbb{Z}) D_{n+1}\right)^{\vee}$ are

$$
\left\langle\check{1}_{e}, \check{1}_{\Lambda^{-1}}, \ldots, \check{1}_{\Lambda^{-(n-2)}}, y 1_{\Lambda^{-(n-1)}}, 1_{\Lambda^{-n}}, \ldots, 1_{\Lambda^{-(2 n-2)}}\right\rangle .
$$

Their bi-degrees are $\overline{\mathcal{Q}}\left(\check{1}_{\Lambda^{-k}}\right)=-\frac{k}{2 n} \check{1}_{\Lambda^{-k}}, \quad \overline{\mathcal{Q}}\left(\check{1}_{\Lambda^{-k}}\right)=\frac{k}{2 n} \check{1}_{\Lambda^{-k}}$ for $k \in$ $\{0,1, \ldots, 2 n-1\} \backslash\{n-1\}$ and $\check{\mathcal{Q}}\left(y \check{1}_{\Lambda^{-(n-1)}}\right)=-\frac{n-1}{2 n} y \check{1}_{\Lambda^{-(n-1)}}$, $\overline{\mathcal{Q}}\left(y \check{1}_{\Lambda^{-(n-1)}}\right)=$ $\frac{n-1}{2 n} y \check{1}_{\Lambda^{-(n-1)}}$.

This is the spectrum of the $(a, c)$ realization of $A_{2 n}$ and there is a projectively unique maximally non-degenerate $G$-Frobenius structure on the dual whose invariants are the $(a, c)$ realization of $A_{2 n-1}$.

(2) In the case that $\sigma\left(\Lambda^{k}\right) \equiv k \bmod 2$ and $n$ is even, then $\sigma(j) \equiv 1$ and the invariants are

$$
\left\langle\check{1}_{e}, \check{1}_{\Lambda^{-2}}, \ldots, 1_{\Lambda^{-(2 n-2)}}, x^{\frac{n-2}{2}} \check{1}_{\Lambda^{-(2 n-1)}}\right\rangle .
$$

Their bi-degrees are $\check{\mathcal{Q}}\left(\check{1}_{\Lambda^{-2 k}}\right)=-\frac{k}{n} \check{1}_{\Lambda^{-2 k}}, \quad \overline{\mathcal{Q}}\left(\check{1}_{\Lambda^{-2 k}}\right)=\frac{k}{n} \check{1}_{\Lambda^{-2 k}}$ for $k \in$ $\{0,1, \ldots, n-1\}$ and $\overline{\mathcal{Q}}\left(x^{\frac{n-2}{2}} \breve{1}_{\Lambda^{2 n-2}}\right)=-\frac{n-1}{2 n} x^{\frac{n-2}{2}} \breve{1}_{\Lambda^{2 n-2}}, \overline{\mathcal{Q}}\left(x^{\frac{n-2}{2}} \breve{1}_{\Lambda^{-(2 n-1)}}\right)=$ $\frac{n-1}{2 n} \check{x}^{\frac{n-2}{2}} 1_{\Lambda^{-(2 n-1)}}$. This is the spectrum of $D_{n+1}$. Furthermore there is a unique maximally non-degenerate $\mathbb{Z} /(2 n \mathbb{Z})$-Frobenius algebra structure of charge $j$ on $\mathbb{Z} /(\check{2} n \mathbb{Z}) D_{n+1}$ which has as invariants the $(a, c)$-realization of $D_{n+1}$.

So for $n$ even, $D_{n+1}$ is self-dual with the choice of non-trivial $\sigma$.

(3) In the case that $\sigma\left(\Lambda^{k}\right) \equiv k \bmod 2$ and $n$ is odd, then $\sigma(j) \equiv 0$ and the invariants are

$$
\left\langle\check{1}_{\Lambda^{-1}}, \check{1}_{\Lambda^{-3}}, \ldots, 1_{\Lambda^{-(2 n-3)}}, x^{\frac{n-1}{2}} \check{1}_{\Lambda^{-(n-1)}}\right\rangle .
$$

Their bi-degrees are $\check{\mathcal{Q}}\left(\check{1}_{\Lambda^{-(2 k+1)}}\right)=-\frac{2 k+1}{2 n} \check{1}_{\Lambda^{-(2 k+1)}}, \overline{\mathcal{Q}}\left(\check{1}_{\Lambda^{-2 k}}\right)=\frac{2 k+1}{n} \check{1}_{\Lambda^{-(2 k+1)}}$ for $k \in\{0,1, \ldots, n-1\}$ and $\breve{\mathcal{Q}}\left(x^{\frac{n-1}{2}} \check{1}_{\Lambda^{n-2}}\right)=-\frac{n-1}{2 n} x^{\frac{n-1}{2}} \check{1}_{\Lambda^{n-2}}, \overline{\mathcal{Q}}\left(x^{\frac{n-2}{2}} \check{1}_{\Lambda^{2 n-2}}\right)=$ $\frac{n-1}{2 n}$.

This case is non $G$-Euler and we see that there is no Frobenius algebra structure on the invariants, since the unit is missing. This means that the prospective unit $\breve{1}_{e}$ is not invariant and there is not even a degenerate G-Frobenius algebra structure on the dual.

4.7. The case of $D_{n+1}$ with the symmetry group $\mathbb{Z} / n \mathbb{Z}$. Let $\mathbb{Z} / n \mathbb{Z} \subset \mathbb{Z} /(2 n \mathbb{Z})$ be the subgroup of even powers $\mathbb{Z} / n \mathbb{Z}=\left\langle\Lambda^{2 k}\right\rangle$.

Remark 4.12. Notice that this subgroup is Euler if and only if $n$ is odd. Also in this case $G_{\max } \neq\langle j\rangle$ and $\mathbb{Z} / n \mathbb{Z} \simeq\langle j\rangle$.

Since most calculations are obtained via restriction from those of the previous section, we handle both the $G$-Frobenius algebras and the duals at the same time.

4.7.1. The bi-gradings. The calculations above for the bi-grading for the $G-$ Frobenius algebra and its dual just restrict to sectors corresponding to the subgroup $\mathbb{Z} / n \mathbb{Z}$.

4.7.2. The $D(k[G])$-modules. Since $\mathbb{Z} / n \mathbb{Z}$ is a cyclic group the discrete-torsion bi-character is trivial: $\epsilon \equiv 1$. In the case that $n$ is odd, there is only one choice $\sigma \equiv 0$. In the case that $n$ is even, there are two choices for $\sigma: \sigma \equiv 0$ and $\sigma\left(\Lambda^{2 k}\right) \equiv k$ mod 2 and In the first case the resulting structure is quasi-Euler, while in the second case it is not. 
Proposition 4.15. For $n$ even and any choice of $\sigma$ the invariants of the resulting $D(k[G])$ module on $\mathbb{Z} / n \mathbb{Z} D_{n+1}$ are two dimensional and are generated by $\left\langle 1_{e}, x^{\frac{n}{2}}\right\rangle$. There is a projectively unique Frobenius structure for the invariants which is the structure of the Frobenius algebra $I_{2}(4)$.

If $n$ is odd, the invariants of $\mathbb{Z} / n \mathbb{Z} D_{n+1}$ are one dimensional and generated by $1_{e}$. Hence they are isomorphic to $A_{1}$ as Frobenius algebras.

In the case that $n$ is even, for the choice of $\sigma \equiv 0$ the resulting $D(k[G])$ module structure on the dual $\mathbb{Z} / n \check{\mathbb{Z}} D_{n+1}$ has invariants

$$
\left\langle\check{1}_{e}, \check{1}_{\Lambda^{-2}}, \ldots, \check{1}_{\Lambda^{-(2 n-2)}}\right\rangle
$$

Their bi-grading is consistent with the $(a, c)$ realization of $B_{n}$ and the respective Frobenius algebra structure is compatible with the group grading. and there is a degenerate $G$-Frobenius structure of charge $j$ on $\mathbb{Z} /(2 n \mathbb{Z})$ restriction to $\mathbb{Z} / 2 \mathbb{Z}$ is the dual $\mathbb{Z} / n \mathbb{Z} D_{n+1}$ and has as invariants precisely the $(a, c)$ realization of $B_{n}$.

In the case that $n$ is odd and, the invariants are:

$$
\left\langle\check{1}_{e}, \check{1}_{\Lambda^{-2}}, \ldots, \check{1}_{\Lambda^{-(n-3)}}, x^{\frac{n-1}{2}} 1_{\Lambda^{-(n-1)}}, y 1_{\Lambda^{-(n-1)}}, 1_{\Lambda^{-(n+1)}}, \ldots, 1_{\Lambda^{-(2 n-2)}}\right\rangle .
$$

with bi-degrees matching the $(a, c)$ realization of $D_{n+1}$ and there a degenerate $G$ Frobenius structure of charge $j$ whose invariants are precisely the $(a, c)$ realization of $D_{n+1}$.

So in the case of $n$ odd $D_{n+1}$ is mirror self dual with respect to the orbifolding by the symmetry group generated by the grading operator

4.8. $D_{n}$ with the symmetry group $\mathbb{Z} / 2 \mathbb{Z}$. In this subsection, we restrict the action of $G_{\max }=\mathbb{Z} /(2 n \mathbb{Z})$ to the subgroup $\mathbb{Z} / 2 \mathbb{Z} \subset \mathbb{Z} /(2 n \mathbb{Z})$ generated by $\Lambda^{n}=$ : -1 .

4.8.1. The algebras $\mathbb{Z} / 2 \mathbb{Z} D_{n+1}$. There are two twisted sectors which as $k$ modules are

$$
\begin{gathered}
A_{e}=D_{n+1}, A_{-1}=A_{n-1} \\
\phi_{-1,-1}=(-1)^{\sigma(-1)+1}
\end{gathered}
$$

There are two choices for $\sigma, \sigma(-1) \equiv 0$ or $\sigma(1) \equiv 1$. The first choice always yields a quasi-Euler $\mathbb{Z} / 2 \mathbb{Z}$ Frobenius algebra, while the latter choice is quasi-Euler only in the case of $n$ odd.

The bi-grading and $\mathbb{Z} / 2 \mathbb{Z}$ action can be read off from the tables in the previous section.

After fixing $\sigma$ there is a unique $\mathbb{Z} / 2 \mathbb{Z}$-Frobenius algebra structure [Ka3, Ka4] which is given by

$$
1_{-1} \circ 1_{-1}=x
$$

4.8.2. The duals. For the dual both $\check{A}_{e}$ and $\check{A}_{-1}$ are one-dimensional and have degrees $(0,0),(-1 / 2,1 / 2)$. Since this is at most a quasi-Euler we cannot pull back the metric, but there is projectively only one metric compatible with the group grading.

The action is given by

$$
\check{\varphi}_{-1,1}=1 \quad \check{\varphi}_{-1,1}=(-1)^{\sigma(-1)}
$$


Proposition 4.16. In the case that $\sigma(-1) \equiv 0$, the invariants are given by

$$
\left\langle 1, x, \ldots, x^{n-1}\right\rangle
$$

The bi-grading and metric and multiplication are commensurate with those of the $(c, c)$ realization of $B_{n}$. The dual algebra has a projectively unique Frobenius algebra structure compatible with the bi-grading that is isomorphic to $I_{2}(4)$ and the invariants are $A_{1}$.

In the case that $n$ is odd and $\sigma(-1)=-1$, the invariants are

$$
\left\langle 1, x, \ldots, x^{n-1}, 1_{-1}, x 1_{-1}, \ldots, x^{n-1} 1_{-1}\right\rangle
$$

The algebra of invariants is isomorphic to the $(c, c)$ realization of $A_{2 n-1}$ as a bigraded Frobenius algebra.

The dual algebra affords the structure of the $(a, c)$ realization of $I_{2}(4)$ with trivial $\mathbb{Z} / 2 \mathbb{Z}$ action.

4.8.3. The case of $D_{4}$ and the relation to $G_{2}$. In the case $n=4$ the maximal symmetry group is $\left\langle\Lambda, \frac{1}{2}\left(\begin{array}{cc}-1 & i \\ -3 i & 1\end{array}\right)\right\rangle \subset G L(2, \mathbb{C})$

Let

$$
j=\left(\begin{array}{cc}
\zeta_{3} & 0 \\
0 & \zeta_{3}
\end{array}\right) a=\left(\begin{array}{cc}
1 & 0 \\
0 & -1
\end{array}\right), b=\frac{1}{2}\left(\begin{array}{cc}
-1 & i \\
-3 i & 1
\end{array}\right)
$$

Then $a^{2}=b^{2}=i d, a b a=b a b$ and $\langle a, b\rangle \simeq \mathbb{S}_{3}$ the symmetric group on three elements. Also $\Lambda=a j$ and $\langle\Lambda\rangle=\mathbb{Z} / 6 \mathbb{Z}=\mathbb{Z} / 3 \mathbb{Z} \times \mathbb{Z} / 2 \mathbb{Z}$. Finally $G_{\max }=\mathbb{Z} / 3 \mathbb{Z} \times \mathbb{S}_{3}$.

We do not want to present the full calculation, which is quite involved, but note that the $G$-Frobenius algebra for $D_{4} / \mathbb{S}_{3}$ is given as a $k$-module by $A_{e}=$ $D_{4}, A_{a}=A_{b}=A_{a b a}=A_{2}, A_{a b}=A_{b a}=A_{1}$. There are three conjugacy classes and the invariants are $1, x^{2}, 1_{a b} \pm 1_{b a}$ where the sign is + if one uses $\sigma \equiv 0$ or - if $\sigma(g) \equiv \operatorname{length}(g)$.

For the invariance of $x^{2}$ notice that in the Milnor ring without using an isomorphism $y^{2}=-3 x^{2}$ and thus $\left(\frac{1}{2}(-1+i y)\right)^{2}=\frac{1}{4} x^{2}-\frac{1}{4} y^{2}+\frac{1}{2} i x y \equiv \frac{1}{4} x^{2}-\frac{-3}{4} y^{2}=x^{2}$.

In the case of the group $\mathbb{Z} / 3 \mathbb{Z}$ generated by $a b$ there the $k$-module is given by $A_{e}=D_{4}, A_{a b}=A_{b a}=A_{1}$ and the invariants are $1, x^{2}, 1_{a b}, 1_{b a}$.

Lemma 4.6. The invariants in the untwisted sector of $D_{4} /(\mathbb{Z} / 3 \mathbb{Z})$ and $D_{4} / \mathbb{S}_{3}$ are isomorphic to $G_{2}$ as graded Frobenius algebras.

4.9. The case $E_{7}$. Recall that for $E_{7}: x^{3}+x y^{3}$, we have the following degrees $q_{1}=q_{x}=\frac{1}{3}, q_{2}=q_{y}=\frac{2}{9}, d=\frac{8}{9}$.

Fix $\zeta_{9}:=\exp \left(2 \pi i \frac{1}{9}\right)$ then the $E_{7}$ singularity has the exponential grading operator $J=\exp (2 \pi i Q)$

$$
J=\left(\begin{array}{cc}
\zeta_{9}^{3} & 0 \\
0 & \zeta_{9}^{2}
\end{array}\right)
$$

This operator generates a subgroup $\langle J\rangle \subset G L(n, \mathbb{C})$ which is isomorphic to $\mathbb{Z} / 9 \mathbb{Z}$. We fix a generator $j$ of $\mathbb{Z} / 9 \mathbb{Z}$ and regard the representation $\rho: \mathbb{Z} / 9 \mathbb{Z} \rightarrow G L(n, \mathbb{C})$ given by $\rho(j)=J$.

This is also the maximal symmetry group $G_{\max }=\langle\Lambda\rangle$

$$
\Lambda=\left(\begin{array}{cc}
\zeta_{9}^{3} & 0 \\
0 & \zeta_{9}^{-1}
\end{array}\right)
$$


and $J=\Lambda^{7}$.

4.9.1. The $\mathbb{Z} / 9 \mathbb{Z}$ - graded $k$-module $\mathbb{Z} / 9 \mathbb{Z} M_{f}$. The representation is given by

$$
\rho\left(j^{i}\right)=\left(\begin{array}{cc}
\zeta_{9}^{3 i} & 0 \\
0 & \zeta_{9}^{2 i}
\end{array}\right)
$$

\begin{tabular}{c|c|r|r|r|r|r|r|r|r}
$g \in \mathbb{Z} / 9 \mathbb{Z}$ & $f_{g}$ & $M_{f_{g}}$ & $d_{g}$ & $\nu_{1}(g)$ & $\nu_{2}(g)$ & $s_{g}^{+}$ & $s_{g}^{-}$ & $s_{g}$ & $\bar{s}_{g}$ \\
\hline$e=j^{0}$ & $x^{3}+x y^{3}$ & $E_{7}$ & $\frac{8}{9}$ & 0 & 0 & 0 & 0 & 0 & 0 \\
$j^{1}$ & 0 & $A_{1}$ & 0 & $\frac{1}{3}$ & $\frac{2}{9}$ & $\frac{8}{9}$ & $-\frac{8}{9}$ & 0 & $\frac{8}{9}$ \\
$j^{2}$ & 0 & $A_{1}$ & 0 & $\frac{2}{3}$ & $\frac{4}{9}$ & $\frac{8}{9}$ & $\frac{2}{9}$ & $\frac{5}{9}$ & $\frac{1}{3}$ \\
$j^{3}$ & $x^{3}$ & $A_{2}$ & $\frac{1}{3}$ & 0 & $\frac{2}{3}$ & $\frac{5}{9}$ & $\frac{1}{3}$ & $\frac{4}{9}$ & $\frac{1}{9}$ \\
$j^{4}$ & 0 & $A_{1}$ & 0 & $\frac{1}{3}$ & $\frac{8}{9}$ & $\frac{8}{9}$ & $\frac{4}{9}$ & $\frac{2}{3}$ & $\frac{2}{9}$ \\
$j^{5}$ & 0 & $A_{1}$ & 0 & $\frac{2}{3}$ & $\frac{1}{9}$ & $\frac{8}{9}$ & $-\frac{4}{9}$ & $\frac{2}{9}$ & $\frac{2}{3}$ \\
$j^{6}$ & $x^{3}$ & $A_{2}$ & $\frac{1}{3}$ & 0 & $\frac{1}{3}$ & $\frac{5}{9}$ & $-\frac{1}{3}$ & $\frac{1}{9}$ & $\frac{4}{9}$ \\
$j^{7}$ & 0 & $A_{1}$ & 0 & $\frac{1}{3}$ & $\frac{5}{9}$ & $\frac{8}{9}$ & $-\frac{2}{9}$ & $\frac{1}{3}$ & $\frac{5}{9}$ \\
$j^{8}$ & 0 & $A_{1}$ & 0 & $\frac{2}{3}$ & $\frac{7}{9}$ & $\frac{8}{9}$ & $\frac{8}{9}$ & $\frac{8}{9}$ & 0
\end{tabular}

Lemma 4.7. The elements of bi-degree $(q, q)$ of $\mathbb{Z} / 9 \mathbb{Z} E_{7}$ are exactly the elements in the untwisted sector $A_{e}$.

4.9.2. The $G$-action. For $\mathbb{Z} / 9 \mathbb{Z} \epsilon \equiv 1$ and $\sigma \equiv 0$, so the $G$-action is given by

$$
\varphi_{j^{i}, j^{k}}= \begin{cases}1 & \text { if } k=0 \\ \zeta_{9}^{-2 i} & \text { if } k \in\{3,6\} \\ \zeta_{9}^{-5 i} & \text { else }\end{cases}
$$

and the character is

$$
\chi\left(j^{i}\right)=\zeta_{9}^{5 i}
$$

Lemma 4.8. The $\mathbb{Z} / 9 \mathbb{Z}$ invariants of the only compatible $D(k[\mathbb{Z} / 9 \mathbb{Z}]$ module structure is given by the unit $1_{e}$.

4.9.3. The dual bi-grading. The dual grading is given by

\begin{tabular}{c|c|c|c|c|c|c|c|c|c} 
& 0 & 1 & 2 & 3 & 4 & 5 & 6 & 7 & 8 \\
\hline$\check{s}_{j^{i}}$ & 0 & $-\frac{8}{9}$ & $-\frac{8}{9}$ & $-\frac{1}{3}$ & $-\frac{4}{9}$ & $-\frac{2}{9}$ & $-\frac{2}{3}$ & $-\frac{7}{9}$ & $-\frac{5}{9}$ \\
$\check{s}_{j^{i}}$ & 0 & 0 & $\frac{8}{9}$ & $\frac{1}{3}$ & $\frac{1}{9}$ & $\frac{2}{9}$ & $\frac{2}{3}$ & $\frac{4}{9}$ & $\frac{5}{9}$
\end{tabular}

The elements of bi-degree $(-q, q)$ are

$$
\left\langle\check{1}_{e}, y^{2} \check{1}_{j}, \check{1}_{j^{2}}, \check{1}_{j^{3}}, \check{1}_{j^{5}}, \check{1}_{j^{6}}, \check{1}_{j^{8}}\right\rangle
$$

4.9.4. The dual $\mathbb{Z} / 9 \mathbb{Z}$ action. The dual $\mathbb{Z} / 9 \mathbb{Z}$ action is given by

$$
\check{\varphi}_{j^{i}, j^{k}}= \begin{cases}\zeta_{9}^{5 i} & \text { if } k=1 \\ \zeta_{9}^{3 i} & \text { if } k \in\{4,7\} \\ 0 & \text { else }\end{cases}
$$


Lemma 4.9. The $\mathbb{Z} / 9 \mathbb{Z}$ invariants of the dual $\mathbb{Z} / 9 \mathbb{Z} E_{7}$ are given by

$$
\left\langle\check{1}_{e}, y^{2} \check{1}_{j}, \check{1}_{j^{2}}, \check{1}_{j^{3}}, \check{1}_{j^{5}}, \check{1}_{j^{6}}, \check{1}_{j^{8}}\right\rangle
$$

they are all of diagonal bi-degree, and their degrees are

$$
(0,0),\left(-\frac{4}{9}, \frac{4}{9}\right),\left(\frac{-8}{9}, \frac{8}{9}\right),\left(-\frac{1}{3}, \frac{1}{3}\right),\left(-\frac{2}{9}, \frac{2}{9}\right),\left(-\frac{2}{3}, \frac{2}{3}\right),\left(-\frac{5}{9}, \frac{5}{9}\right)
$$

The pairing and bi-grading and the group grading are commensurate with that of the anti-chiral realization of $E_{7}$ under the association $\check{1}_{e} \mapsto 1, \breve{1}_{j} \mapsto y^{2}, \breve{1}_{j^{2}} \mapsto$ $x^{2} y, \check{1}_{j^{3}} \mapsto x, \check{1}_{j^{5}} \mapsto y, \check{1}_{j^{6}} \mapsto x^{2}, \check{1}_{j^{8}} \mapsto x y$, so that $E_{7}$ is self dual.

Again by inspecting the grading and group grading

Proposition 4.17. There is a unique maximally degenerate G-Frobenius structure of charge $j$ on $\mathbb{Z} / 9 \mathbb{Z} E_{7}$ whose invariants form the $(a, c)$ realization of $E_{7}$. Hence $\left(\mathbb{Z} / 9 \mathbb{Z} E_{7}\right)^{\mathbb{Z} / 9 \mathbb{Z}}$ is the mirror dual to $E_{7}$.

4.10. The case $P_{8}$. We would briefly digress to singularities of higher modularity. The first singularity of this type is $P_{8}=x^{3}+y^{3}+z^{3}-a x y z$ with $a^{3}+27 \neq 0$. The Milnor ring of this singularity is given generated by $\langle 1, x, y, z, x y, y z, x z, x y z\rangle$. It is quasi homogenous of degrees $q_{x}=q_{y}=q_{z}=\frac{1}{3}$ and $d=1$.

This singularity is not self-dual. Moreover in the case that $a \neq 0$ there is no symmetry group which has only $A_{1}$ as invariants of the $G$-Frobenius algebra, since the term $x y z$ always has to remain invariant, so it is impossible for this a $G$-Frobenius algebra built from this dual singularity to be mirror-dual for any orbifolding group to another singularity. Also the invariants cease to have the diagonal $(q, q)$ or anti-diagonal $(-q, q)$ grading.

Let us calculate $P_{8} / \Gamma$ for the group $\gamma$ generated by the grading operator $J=$ $\operatorname{diag}\left(\zeta_{3}, \zeta_{3}, \zeta_{3}\right)$. There are two one-dimensional twisted sectors.

The shifts for the twisted sectors $i=1,2$ are $s_{J}=0, \bar{s}_{J}=1 ; s_{J^{2}}=1, \bar{s}_{J^{2}}=0$ Since $\operatorname{det}\left(J^{i}\right)=1$ and necessarily $\sigma \equiv 0, \epsilon \equiv 1$ all elements in the twisted sector are invariant. In total the invariant elements are

$$
1, x y z, 1_{J}, 1_{J^{2}} \text { of degrees }(0,0),(1,1),(1,0) \text { and }(0,1)
$$

For the dual, the action does not change since $\sigma \equiv 0$ and hence $\chi \equiv 1$ and we obtain the same invariants, only with a shifted group grading.

Remark 4.13. Notice that the spectrum is such that is looks like the Hodge diamond of manifold.

Proposition 4.18. The $G$-Euler $G$-Frobenius algebra $\mathbb{Z} / 3 \mathbb{Z} P_{8}$ is mirror self-dual: $\left(\mathbb{Z} / 3 \mathbb{Z} P_{8}\right)^{\mathbb{Z} / 3 Z} \simeq\left(\left(\mathbb{Z} / 3 \mathbb{Z} P_{8}\right)^{\vee}\right)^{\mathbb{Z} / 3 Z}$.

\section{Remarks on the Relation to SPIN CURVES, the GeOMetry of SINGULARITIES AND FOLDING}

5.1. Remarks on the relation to $r$-spin curves and $A$-models for quasihomogenous polynomials. The $r$-spin curve picture was conceived by Witten as an $A$-model or $\sigma$-Model counterpart for the $A_{r-1}$ Landau-Ginzburg $B$-model $[\mathrm{W}]$. In his construction and the mathematical constructions of [JKV, PV, P] this was achieved. It turns out however, that in the formulation there are two types of behaviors at given marked points called Ramond or Neveu-Schwarz. The appearance of the Ramond case introduces an additional element in the state space, 
which is $n+1$ dimensional in the $A_{n}$ case. If this element appears in a correlation function the value of the correlation function becomes zero. So the algebra is what we called a degenerate Frobenius algebra of degree $j$ if one assigns the group degree $j^{-1}$ to $z$ and identifies the Ramond element with $z^{-n}$.

This is the projectively unique maximally degenerate $G$-Frobenius algebra one obtains from $(p t /(\mathbb{Z} /(n+1) \mathbb{Z}))^{\vee}[\mathrm{Ka} 3]$. If one considers $A_{1}$ as the $(a, c)$ ring of $A_{n}$ then by self duality of $A_{n}$ one could expect that $\left.\left(\left(A_{1}=p t\right) / \mathbb{Z} /(n+1) \mathbb{Z}\right)^{\vee}\right)^{\mathbb{Z} /(n+1) \mathbb{Z}=}$ $A_{n}$ (cf. [Ka3]) which is indeed the structure found above. In this interpretation the bi-grading is however not straightforward, although the grading could be recovered from the $q_{i}$ and $\nu_{i}$ by considering the action of $\mathbb{Z} /(n+1) \mathbb{Z}$ on $\mathbb{C}$ by roots of unity.

It would be desirable to consider not only this altered version of our duality applied to the $(a, c)$ ring and not the $(c, c)$ ring, but to see it directly on the $(c, c)$ side.

For this, we would like to give another interpretation of our previous remark on the $A_{r-1}$ model. The equation here which is mimicked in the case of spin curves is $z^{r}=0$ only that in the spin-curve picture $\mathcal{L}^{\otimes r} \simeq \omega$ (twisted) (Here $\omega$ (twisted) is a suitably twisted version of the canonical line bundle on the curve). The fact that the Ramond sector is zero in all the correlation functions can be taken to mean that it is in fact zero. In other words it appears only as a degenerate state and as we project to the invariants the equation $z^{r}=0$ is implemented. In algebraic terms for $A_{r-1}$, first one considers $R:=\mathbb{C} /\left(z^{r}\right)$ and then $R /\left(z^{r-1}\right)$. The first quotient is inherent in the spin picture in the periodicity with respect to $\omega$ (twisted $) \simeq \mathcal{L}^{\otimes r}$.

Now our degenerate Ramond sector is in fact $n$ dimensional for $A_{n}$ and not one dimensional. Here one should remark that for the construction of of an operad in the Ramond case, one would actually have to fix a choice of isomorphism of the line bundle with $\omega$ (twisted). The space of choices for this isomorphism is a principal $\mathbb{Z} /(n+1) \mathbb{Z}$ space and thus if one includes this data in the moduli problem the state space for the Ramond sector becomes $n+1$ dimensional for $A_{n}[\mathrm{~J}]$. So indeed the Ramond sector seems to be intrinsically higher dimensional. The fact that the dimension is not $n$, but $n+1$ dimensional can be understood by the reasoning before. In our description the singularity in this sector is the singularity $A_{n}$. In the Milnor ring interpretation this produces a Frobenius algebra which has $n$ states. In the spin-representation as discussed above one would expect $n+1$ states, one of which is degenerate.

The musings on this subject are at the moment only on the level of the undeformed algebra, but we hope to make them into more solid statements.

There is a straightforward way to build a spin curve like picture for any quasihomogenous polynomial $f$. For this one considers a line bundle $\mathcal{L}_{i}$ for each of the variables $z_{i}$ and imposes the equations obtained by substituting the line bundles $\mathcal{L}_{i}$ into the of monomials of the polynomials $f$ and equates these expression to $\omega($ twisted $)$. This defines the moduli problem. This approach is being seriously discussed by [FJR]. When the polynomial $f$ is such that is maximal symmetry group $G_{\max }$ is Abelian and each variable appears by itself, the corresponding virtual fundamental class is constructed in [FJR]. The hope is to be able to lift these conditions $[\mathrm{J}]$. We would like to point out that the condition on the variables appearing alone in a monomial ensures that $\mathbb{C}\left[\left[z_{i}\right]\right] /\left(m_{j}\right)$ is finite dimensional. Here the $m_{j}$ are the monomials of $f$. 
Further evidence for our interpretation of "Landau-Ginzburg $A$-models" arises from these constructions. For each element $g \in G_{\max }$ there are again two types of behaviors at the marked point which are either of Ramond or of Neveu-Schwarz type. The Ramond means that the isotropy at a marked point is not the full symmetry group while in the Neveu-Schwarz case it is.

Again to turn the resulting moduli spaces into operads it is necessary to include additional data for the Ramond case which is isomorphic to the reduced symmetry group of $f_{g}[\mathrm{~J}]$.

Conjecture 5.1. We conjecture that the Neveu-Schwarz sectors are in 1-1 correspondence with the one-dimensional twisted sectors and the Ramond sectors are in one-one correspondence with the sectors that are more than one-dimensional, i.e. $Q_{f_{g}} \neq \mathbb{C}$.

This conjecture has been checked against the preliminary results of [FJR].

Conjecture 5.2. We expect that the non-degenerate part of the cohomological Field theory described by a quasi-homogenous polynomial is the deformation of the Frobenius algebra of the invariants of $\left(G_{\max } M_{f}\right)^{\vee}$. Moreover, we expect that the behavior of the correlation functions is modelled by the deformations of a degenerate $G$-Frobenius of charge $j$ given by $\left(G_{\max } M_{f}\right)^{\vee}$, possibly adding more degenerate elements. More precisely, let $m_{j}$ be the monomials of $f$ and $q_{i}=\frac{1}{n_{i}}$ be the quasihomogenous degrees of the $z_{i}$. In the case that the ring $\hat{M}_{f_{g}}:=\mathcal{O} /\left(m_{f_{g}, j}\right)$ is finite dimensional, the extra elements should correspond to the extension of basis from $M_{f_{g}}$ to $\hat{M}_{f_{g}}$ - for each higher-dimensional sector.

Our calculations predict that this procedure yields the right result in the case of Pham singularities with coprime powers, such as $E_{6}$ and $E_{8}$ and indeed this is true by taking tensor products of spin-curves [JKV2].

5.2. Orbifolding and the geometry of singularities with symmetries. There is a relationship of our constructions of $G$-Frobenius manifolds for a singularity $f$ and the Ramond state space of [Ka3] (not to be confused with the Ramond notation for spin-curves) and classic singularity theory.

For a singularity there are classically two objects which are studied, one is the Milnor ring $M_{f}$ which also provides a basis for the minversal unfolding which can be written as

$$
F:\left(\mathbb{C}^{n+1} \times M_{f}, 0\right) \rightarrow(\mathbb{C}, 0)
$$

This fact affords an extension by the choice of a primitive form [S] to a construction of Frobenius manifold on the flat space $M_{f}[\mathrm{Du}]$.

The other object of interest obtained from the Milnor fibration which is given by

$$
f:\left(\mathbb{C}^{n+1}, 0\right) \rightarrow(\mathbb{C}, 0)
$$

which gives a local fibration on $\mathbb{C}-0$. The fibers are bouquets of spheres and the Betti number of the middle dimensional cohomology of the fibers is also $\mu$. The isomorphism between $M_{f}$ and $H:=H^{n}\left(F_{*}, \mathbb{C}\right)$ can be given by a choice of primitive form. Here $F_{*}$ denotes a generic fiber.

Now suppose $G \subset G L(n+1, \mathbb{C})$ is a group of symmetries. This will act on the total space of the Milnor fibration and trivially on base and thus there is an induced action on $H$. 
Let det be the one dimensional representation of $G$ given by the determinant.

The main result of $[\mathrm{Wa}]$ is

Theorem 5.1 ([Wa]). In the situation described above the $\mathbb{C}[G]$ modules $H$ and $M_{f} \otimes$ det are isomorphic.

This infers that while the untwisted sector of the $G$-Frobenius algebra is isomorphic as a $\mathbb{C}[G]$-module to $M_{f}$ the untwisted sector of the Ramond state space is isomorphic as a $\mathbb{C}[G]$-module to $H$. This untwisted Ramond sector corresponds to the $j$ twisted sector of the dual.

In exactly the case that the symmetries generate a Coxeter group $G$ the quotient of $\mathbb{C}^{n+1}$ by $G$ is smooth: $\mathbb{C}^{n+1} / G \simeq \mathbb{C}^{n+1}$. In this situation, one can regard the germ $f_{G}$ on the quotient. Let $\mu_{G}$ denote the Milnor number of $f_{G}$ and $\mu_{g}$ those of $f_{g}:=\left.f\right|_{\operatorname{Fix}(G)}$. Here we need to assume that this restriction is again an isolated singularity which is automatic in the quasi-homogenous case. Also fix $d_{g}=\operatorname{codim}(\operatorname{Fix}(g))$

The results of $[\mathrm{Wa}]$ are

$$
\mu_{G}=\frac{1}{|G|} \sum_{g \in G}(-1)^{d_{g}} \mu_{g}
$$

Furthermore in $[\mathrm{Wa}]$ the equivariant Euler-characteristic of the $\mathbb{C}[G]$-modules $M_{f}$ and $H$ is used to compute the Milnor numbers $\mu_{g}$. Let $M=H_{n}\left(F_{*}, \mathbb{C}\right)$ and consider its class $[M]$ in the representation ring of $G$. We can identify this with the ring of class functions and evaluate at elements $g$.

The formula is $[\mathrm{Wa}]$

$$
\mu_{g}=(-1)^{d_{g}}[M](g)
$$

This give a way to compute the invariants of the untwisted Ramond state space which is isomorphic as a $G$-module to the $j$ twisted sector of $\breve{G} M_{f}$.

It is interesting to note that the twisted sectors contribute to this calculation through the equivariant Euler characteristic.

One could adapt these techniques to the restrictions of the singularity to the various fixed point sets and obtain formulas for the dimension of the whole space $\check{M}_{f}$.

5.3. Folding. For the Dynkin diagrams of the simple singularities and more generally for the generalized Dynkin diagrams of $[Z]$, there is an operation known as folding.

In this section we show, that the folding can be described as a non-stringy orbifolding with respect to a group of projective symmetries.

Definition 5.1. A projective symmetry for a singularity $f: \mathbb{C} \rightarrow \mathbb{C}$ with an isolated critical at zero is an element $S \in G L(n, \mathbb{C})$, s.t. $f(S(\mathbf{z}))=\lambda f(\mathbf{z})$ for some $\lambda \in \mathbb{C}$

A projective folding group for a quasi-homogenous singularity $f$ is group $G$ together with a representation of $G$ in $G L(n, \mathbb{C})$ which acts by projective symmetries with the same fixed $\lambda$ and preserves the unique (up to scalar multiples) element of highest degree.

These type of symmetries act on the Milnor ring, since the local $\operatorname{ring} f(\mathbf{z})=0$ is equal to that of $\lambda f(\mathbf{z})=0$. 


\begin{tabular}{c|c|c|c} 
Diagram/group & Folded diagram/group & Folding group & representation \\
$A_{n}$ & $I_{2}(n+1)$ & $\mathbb{Z} /(n-1) \mathbb{Z}$ & $z \mapsto \zeta_{n-1} z$ \\
$A_{2 n-1}$ & $B_{n}$ & $\mathbb{Z} / 2 \mathbb{Z}$ & $z \mapsto-z$ \\
$D_{n+1}$ & $B_{n}$ & $(x, y) \mapsto(x,-y)$ \\
$D_{4}$ & $G_{2}$ & $\mathbb{Z} / 2 \mathbb{Z}^{*}$ & $(x, y) \mapsto(-x,-y)$ \\
$D_{6}$ & $H_{3}$ & $(x, y) \mapsto(-x,-y)$ \\
$E_{6}$ & $F_{4}$ & $(x, y) \mapsto(x,-y)$ \\
$E_{8}$ & $H_{4}$ & $e \times \mathbb{Z} / 3 \mathbb{Z}$ & $(x, y) \mapsto\left(x, \zeta_{3} y\right)$
\end{tabular}

* This is the simplest group. Other folding groups are $\mathbb{Z} / 3 \mathbb{Z}$ and $\mathbb{S}_{3}$ as discussed in $\S 4.8 .3$.

TABLE 3. The Foldings and their projective symmetry groups.

Remark 5.1. For a sum of two singularities $f+g$, the product of two projective symmetry groups for $f$ and $g$ respectively also acts on the Milnor ring $M_{f+g}=$ $M_{f} \otimes M_{g}$

Theorem 5.2. For each of the classical foldings for Coxeter groups there is a group of projective symmetries or a product of two groups of projective symmetries which has as its invariants the Frobenius algebra of the folded graph. The foldings and groups are contained in table 5.3.

Remark 5.2. The utilization of projective symmetries is necessary, since not all foldings can be realized with $\lambda=1$ in particular the element of highest degree transforms in the representation $\operatorname{det}(\rho(g))^{-2}$ (see e.g. [Ka3]) so that the only folding groups with $\lambda=1$ will be those whose determinant lie in \pm 1 . In particular the $\mathbb{Z} / 2 \mathbb{Z}$ foldings of $A_{2 n-1}$ and $D_{n+1}$ yielding as discussed above and also $E_{6}$ to $F_{4}$ can be realized by orbifolding. For $G_{2}$ the folding can be only be obtained via orbifolding by restricting to the classical level, i.e. disregarding the twisted sectors.

Remark 5.3. The folding of $E_{6}$ and $E_{8}$ can also be understood as the tensor products of the folding on the factors. $A_{2} \otimes I_{2}(4)=F_{4}$ and $A_{2} \otimes I_{2}(5)$.

Remark 5.4. Unlike in the case of the operation of symmetries, the group action of projective symmetries does not act on the Milnor fibration fiberwise and hence not obviously on the cohomology bundle. But on the other hand it leaves the central fiber invariant and furthermore acts by homothety on the base via $f(z)=t \mapsto f(z)=\frac{1}{\lambda} t$, so we obtain an equivariant action.

Remark 5.5. The relation of folding to the miniversal unfolding space is known and is given in $[\mathrm{St}]$. In fact the foldings provide submanifolds of Frobenius manifolds or $\mathcal{F}$ manifolds.

Remark 5.6. It would be desirable to extend the theory of $G$-Frobenius algebras to these quotients. In fact it seems to be straightforward to generalize some of the construction of [Ka3] for Jacobian Frobenius algebras with symmetries to those with projective symmetries. Here the twisted sectors would again just be obtained from the function by restriction to the fixed subspace. There is also no obstruction to keeping the grading shifts and dualization process. One would expect to be able to apply this type of orbifolding to the calculations and definition of [Z]. We leave the more careful analysis of this possibility for the future. 


\section{REFERENCES}

[AGLV] V. I. Arnold et al. Singularity theory. I. Translated from the 1988 Russian original by A. Iacob. Reprint of the original English edition from the series Encyclopaedia of Mathematical Sciences [ Dynamical systems. VI, Encyclopaedia Math. Sci., 6, Springer, Berlin, 1993

[CR] W. Chen and Y. Ruan, A new cohomology theory for orbifold. math.AG/0004129. And W. Chen and Y. Ruan, Orbifold Gromov-Witten theory. In A. Adem, J. Morava, and Y. Ruan (eds.), Orbifolds in Mathematics and Physics, Contemp. Math., Amer. Math. Soc., Providence, RI. 310, (2002), 25-85. math.AG/0103156.

[Du] B. Dubrovin, Geometry of 2D topological field theories. in Integrable systems and quantum groups (Montecatini Terme, 1993), Lecture Notes in Math., 1620, Springer, Berlin, 1996, pp. $120-348$.

[FJR] H. Fan, T. Jarvis and Y. Ruan, A Generalization of Spin Orbifold Quantum Cohomology Arising From Quasi-Homogeneous Polynomials. In preparation.

[GP] B. R. Greene, M. R. Plesser, Duality in Calabi-Yau moduli space, Nuclear Phys. B 338 (1990), 15-37.

[IV] K. Intriligator and C. Vafa, Landau-Ginzburg orbifolds, Nuclear Phys. B 339 (1990), 95-120

$[\mathrm{J}]$ T. Jarvis. Private communication.

[JKK] T. Jarvis, R. Kaufmann and T. Kimura, Pointed Admissible $G$-Covers and $G$-equivariant Cohomological Field Theories, Preprint math.AG/0302316. 14 (2003), 573-619.

[JKV] T. Jarvis, T. Kimura and A. Vaintrob. Moduli spaces of higher spin curves and integrable hierarchies Compositio Math. 126 (2001).

[JKV2] T. Jarvis, T. Kimura and A. Vaintrob. Tensor products of Frobenius manifolds and moduli spaces of higher spin curves. Conférence Moshé Flato 1999, Vol. II (Dijon), 145-166, Math. Phys. Stud., 22, Kluwer Acad. Publ., Dordrecht, 2000.

[Ka1] R. M. Kaufmann, The tensor Product in the Theory of Frobenius manifolds, Int. J. of Math. 10 (1999) 159-206.

[Ka2] R. M. Kaufmann, Orbifold Frobenius algebras, cobordisms, and monodromies. In A. Adem, J. Morava, and Y. Ruan (eds.), Orbifolds in Mathematics and Physics, Contemp. Math., Amer. Math. Soc., Providence, RI. 310, (2002), 135-162.

[Ka3] R. M. Kaufmann Orbifolding Frobenius algebras. Int. J. of Math. 14 (2003), 573-619.

[Ka4] R. M. Kaufmann, Second quantized Frobenius algebras, Commun. Math. Phys. to appear.

[Ka5] R. M. Kaufmann. Discrete torsion, symmetric products and the Hilbert scheme. To appear in the conference proceedings of the conference in honor of Yuri Ivanvovich Manin's 65th birtday.

[Ka6] R. M. Kaufmann, The algebra of discrete torsion, Preprint. math.AG/0208081.

[M] Yu. Manin, Frobenius manifolds, quantum cohomology, and moduli spaces. Colloquium Publ., 47, Amer. Math. Soc., Providence, RI, 1999.

[Mo] S. Montgomery. Hopf algebras and their actions on rings. CBMS Regional Conference Series in Mathematics, 82. American Mathematical Society, Providence, RI, 1993.

[P] A. Polishchuk. Witten's top Chern class on the moduli space of higher spin curves. Preprint math.AG/0208112.

[PV] A. Polishchuk and A. Vaintrob. Algebraic construction of Witten's top Chern class. Advances in algebraic geometry motivated by physics (Lowell, MA, 2000), 229-249, Contemp. Math., 276, Amer. Math. Soc., Providence, RI, 2001.

[S] K. Saito. Period mapping associated to a primitive form. Publ. Res. Inst. Math. Sci. Kyoto Univ., 19 (1983), 1131-1264. And

K. Saito. Primitive forms for a universal unfolding of a function with an isolated critical point. Journ. Fac. Sci. Univ. Tokyo, Sec. IA. 28 (1982), 775-792.

[St] I.A.B. Strachan. Frobenius submanifolds. J. Geom. Phys. 38 (2001), no. 3-4, 285-307. And I.A.B. Strachan. Frobenius manifolds and bi-Hamiltonian structures on discriminant hypersurfaces. Integrable systems, topology, and physics (Tokyo, 2000), 251-265, Contemp. Math., 309, Amer. Math. Soc., Providence, RI, 2002

[V] C. Vafa, String vacua and orbifoldized LG models, Modern Phys. Lett. A4 (1989), 1169-1185.

[Wa] C. T. C. Wall, A note on symmetry of singularities, Bull. London Math. Soc.12

[W] E. Witten Algebraic geometry associated with matrix models of two- dimensional gravity. Topological models in modern mathematics (Stony Brook, NY, 1991), Publish or Perish, 
Houston, TX (1993), 235-269. and The N-matrix model and gauged WZW models. Nucl. Phys. B371 (1992), 191- 245.1980), 169-175.

[Z] J.-B. Zuber. Generalized Dynkin diagrams and root systems and their folding. In: Topological field theory, primitive forms and related topics (Kyoto, 1996), 453-493, Progr. Math., 160, Birkhuser Boston, Boston, MA, 1998.

E-mail address: ralphk@mpim-bonn.mpg.de

Oklahoma State University, Department of Mathematics, MS 401, Stillwater OK 74078-1058, USA 